\title{
SYNCHROTRON POLARIZATION IN BLAZARS
}

\author{
HaOCheng Zhang ${ }^{1,2}$, Xuhui Chen ${ }^{3,4}$, AND Markus BötTCheR ${ }^{1,5}$ \\ ${ }^{1}$ Astrophysical Institute, Department of Physics and Astronomy, Ohio University, Athens, OH 45701, USA \\ 2 Theoretical Division, Los Alamos National Laboratory, Los Alamos, NM 87545, USA \\ ${ }^{3}$ Institute of Physics and Astronomy, University of Potsdam, D-14476 Potsdam-Golm, Germany \\ ${ }^{4}$ DESY, Platanenallee 6, D-15738 Zeuthen, Germany \\ ${ }^{5}$ Centre for Space Research, North-West University, Potchefstroom 2531, South Africa \\ Received 2014 January 28; accepted 2014 May 20; published 2014 June 16
}

\begin{abstract}
We present a detailed analysis of time- and energy-dependent synchrotron polarization signatures in a shock-in-jet model for $\gamma$-ray blazars. Our calculations employ a full three-dimensional radiation transfer code, assuming a helical magnetic field throughout the jet. The code considers synchrotron emission from an ordered magnetic field, and takes into account all light-travel-time and other relevant geometric effects, while the relevant synchrotron self-Compton and external Compton effects are handled with the two-dimensional Monte-Carlo/Fokker-Planck (MCFP) code. We consider several possible mechanisms through which a relativistic shock propagating through the jet may affect the jet plasma to produce a synchrotron and high-energy flare. Most plausibly, the shock is expected to lead to a compression of the magnetic field, increasing the toroidal field component and thereby changing the direction of the magnetic field in the region affected by the shock. We find that such a scenario leads to correlated synchrotron + synchrotron-self-Compton flaring, associated with substantial variability in the synchrotron polarization percentage and position angle. Most importantly, this scenario naturally explains large polarization angle rotations by $\gtrsim 180^{\circ}$, as observed in connection with $\gamma$-ray flares in several blazars, without the need for bent or helical jet trajectories or other nonaxisymmetric jet features.
\end{abstract}

Key words: galaxies: active - galaxies: jets - gamma rays: galaxies - radiation mechanisms: non-thermal relativistic processes

Online-only material: color figures

\section{INTRODUCTION}

Blazars are an extreme class of active galactic nuclei. They are known to emit nonthermal-dominated radiation throughout the entire electromagnetic spectrum, from radio frequencies to $\gamma$-rays, and their emission is variable on all timescales, in some extreme cases down to just a few minutes (e.g., Aharonian et al. 2007; Albert et al. 2007). It is generally agreed that the nonthermal radio through optical-UV radiation is synchrotron radiation of ultrarelativistic electrons in localized emission regions which are moving relativistically (with bulk Lorentz factors $\Gamma \gtrsim 10$ ) along the jet. The origin of the high-energy (X-ray through $\gamma$-ray) emission is still controversial. Both leptonic models, in which high-energy radiation is produced by the same relativistic electrons through Compton scattering, and hadronic models, in which $\gamma$-ray emission results from proton synchrotron radiation and emission initiated by photo-pionproduction, are currently still viable (for a review of leptonic and hadronic blazar emission models, see, e.g., Böttcher 2007; Böttcher \& Reimer 2012; Krawczynski et al. 2012).

The radio through optical emission from blazars is also known to be polarized, with polarization percentages ranging from a few to tens of percent, in agreement with a synchrotron origin in a partially ordered magnetic field. Both the polarization percentage and position angle are often highly variable (e.g., D'Arcangelo et al. 2007). The general formalism for calculating synchrotron polarization is well understood (e.g., Westfold 1959), and several authors have demonstrated that the observed range of polarization percentages and the dominant position angle in blazar jets are well explained by synchrotron emission from relativistically moving plasmoids in a jet that contains a helical magnetic field (e.g., Lyutikov et al. 2005; Pushkarev et al.
2005). Recently, also the expected X-ray and $\gamma$-ray polarization signatures in leptonic and hadronic models of blazars have been evaluated by Zhang \& Böttcher (2013), demonstrating that highenergy polarization may serve as a powerful diagnostic between leptonic and hadronic $\gamma$-ray production.

Recent observations of large $\left(\gtrsim 180^{\circ}\right)$ polarization-angle swings that occurred simultaneously with high-energy ( $\gamma$-ray) flaring activity (Marscher et al. 2008, 2010; Abdo et al. 2010b), have been interpreted as additional evidence for a helical magnetic field structure. However, on the theory side, there is currently a disconnect between models focusing on a description of the synchrotron polarization features, and models for the broadband (radio through $\gamma$-ray) spectral energy distributions (SEDs) and variability. Models for the synchrotron polarization percentage and position angle necessarily take into account the detailed geometry of the magnetic field and the angle-dependent synchrotron emissivity and polarization (e.g., Lyutikov et al. 2005), but typically apply a simple, time-independent powerlaw electron spectrum and ignore possible predictions for the resulting high-energy emission. On the other hand, most models for the broadband SEDs and variability employ a chaotic magnetic field, where the synchrotron emissivity is angle-averaged, and any angle dependence of synchrotron and synchrotron-self-Compton emissions (in the co-moving frame of the emission region) is ignored.

An attempt to combine polarization variability simulations with a simultaneous evaluation of the high-energy emission, has recently been published by Marscher (2014). In his turbulent, extreme multi-zone (TEMZ) model, the magnetic field along the jet is assumed to be turbulent (i.e., with no preferred orientation), but as electrons in a small fraction of the jet are accelerated to ultrarelativistic energies when passing through 

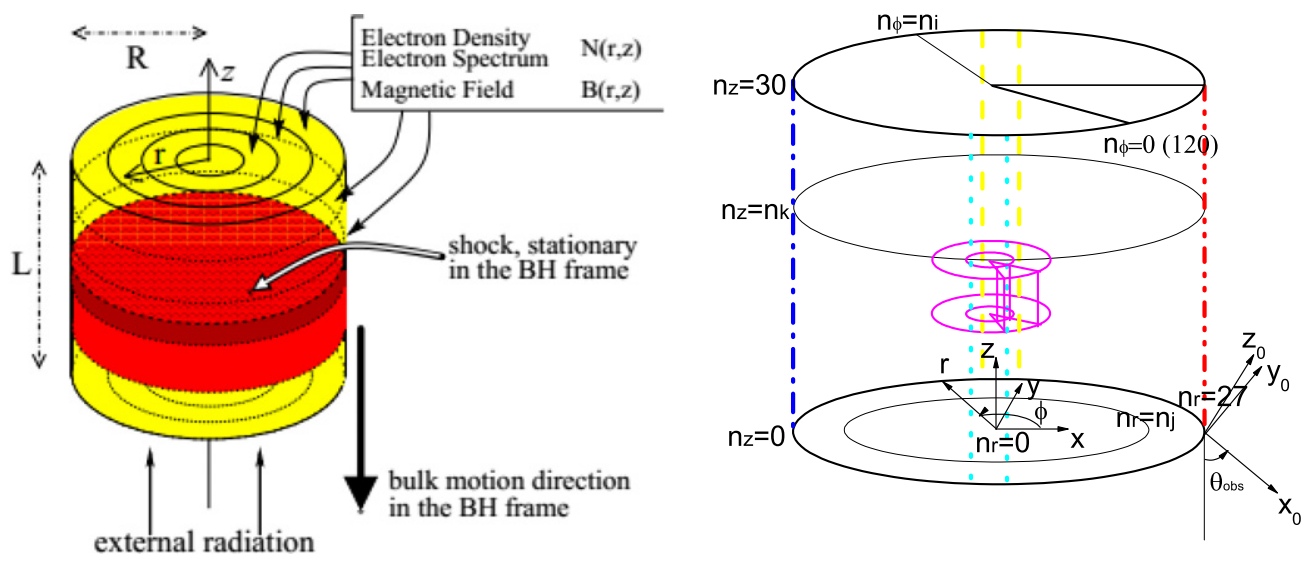

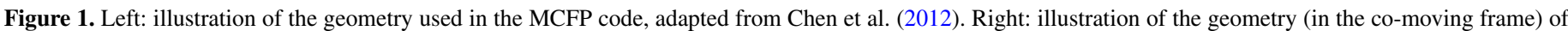

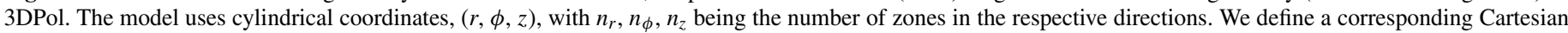

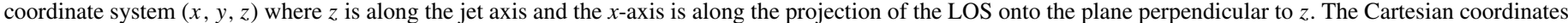

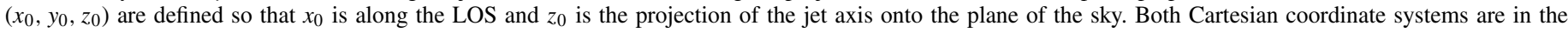

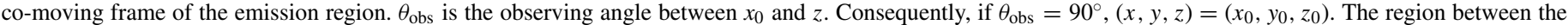

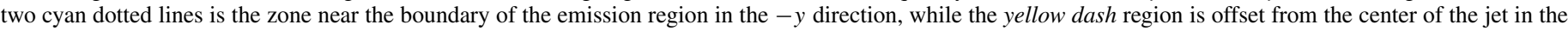

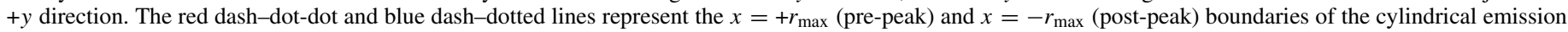
region, respectively. Magenta region represents a near-central zone in the emission blob.

(A color version of this figure is available in the online journal.)

a standing shock, a variable, nonzero percentage of polarization is expected stochastically from the addition of synchrotron radiation from a small number of energized cells with individually homogeneous magnetic fields. While this model does occasionally produce apparent polarization-position-angle rotations, it seems difficult to establish a statistical correlation between $\gamma$-ray flaring activity and position-angle swings in this model. More often, it is argued that an initially chaotic magnetic field is compressed by a shock. As a consequence, in the direction of the line of sight (LOS), the magnetic field may appear ordered locally (e.g., Laing 1980). Alternatively, strong synchrotron polarization may result in a model in which the emission region moves in a helical trajectory (e.g., Villata \& Raiteri 1999) guided by a very strong large-scale magnetic field, so that the magnetic field inside the emission region is very ordered. Helical magnetic fields may be a natural consequence of the rotation of the central black hole and its accretion disk together with the jet outflow (e.g., Contopoulos 1994), or alternately, may arise when an initially toroidal magnetic field is modified by a poloidal magnetic field generated by relativistic shear along the jet (Aloy et al. 2000). In this case, the polarization-percentage and position-angle changes can be associated with the motion of the emission region.

In this paper, we investigate the synchrotron-polarization and high-energy emission signatures from a shock-in-jet model, in which the un-shocked jet is pervaded a helical magnetic field. As the shock moves along the jet, it accelerates particles to ultrarelativistic energies. We consider separately several potential mechanisms through which flaring activity may arise in such a scenario, including the amplification of the toroidal magneticfield component. For the purpose of our simulations, we will employ the time-dependent two-dimensional (2D) radiation transfer model developed by Chen et al. (2011, 2012). This model assumes an axisymmetric, cylindrical geometry for the emission region, and uses a locally isotropic Fokker-Planck equation to evolve the electron distributions. The latest development of this code includes a helical magnetic field structure to replace the original chaotic structure (with angle-averaged emissivities), which makes the evaluation of synchrotron polarization possi- ble. However, this evaluation of polarization requires treatment of synchrotron emission in full three-dimensional (3D) geometry. Since there is a large number of free parameters in this model, we will here focus on a general parameter study, simulating and comparing the polarization patterns for different possible flaring scenarios, rather than fit the observed data directly. In a future paper, we plan to combine MHD simulations with this code in order to constrain the free parameters pertaining to changes in the magnetic-field configuration, and fit the data directly. We will describe the code setup in Section 2, compare different scenarios in Section 3, and discuss the results in Section 4.

\section{CODE SETUP}

In this section, we will first give a brief review of the $2 \mathrm{D}$ radiation transfer model by Chen et al. (2011, 2012), then introduce the $3 \mathrm{D}$ polarization code setup and compare its result with that of the $2 \mathrm{D}$ code.

\subsection{D Monte-Carlo/Fokker-Planck (MCFP) Code}

The code of Chen et al. $(2011,2012)$ assumes an axisymmetric cylindrical geometry for the emission jet, which is further divided evenly into zones in radial and longitudinal directions (Figure 1). The plasma moves relativistically along the jet, which is pervaded by a purely helical magnetic field, and encounters a flat stationary shock. In the comoving frame of the emission region, the shock will temporarily change the plasma conditions as it passes through the jet plasma, and hence generate a flare. In this paper we consider four parameter changes that may characterize the effect of the shock: (1) amplification of the toroidal component of the magnetic field; (2) increase of the total magnetic field strength; (3) shortening of the acceleration timescale of the nonthermal electrons; and (4) injection of additional nonthermal electrons. The model uses a locally isotropic Fokker-Planck equation to evolve the electron distributions in each zone and applies the Monte-Carlo method to track the photons. Hence, the combined scheme is referred to as a Monte-Carlo/Fokker-Planck (MCFP) scheme. Within the 

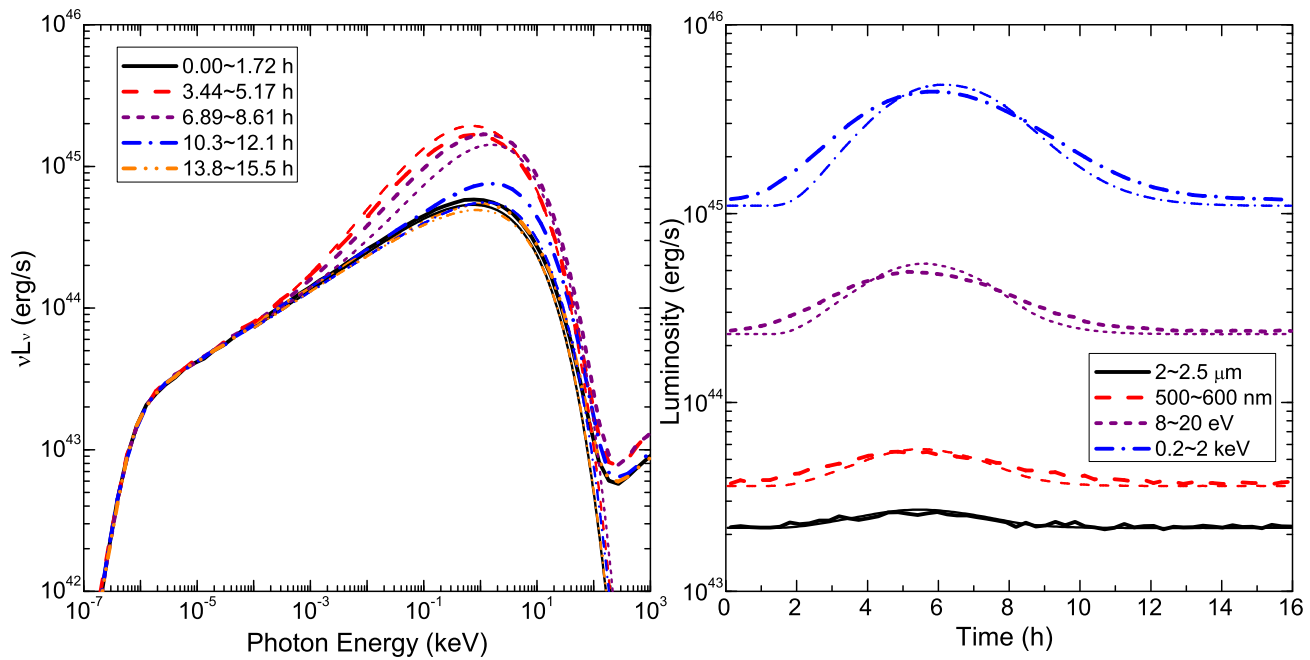

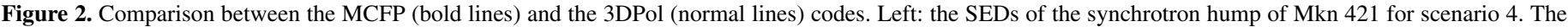
time bins are chosen the same for both codes. Right: the light curves of Mkn 421 for scenario 4 . The energy bins are identical as well.

(A color version of this figure is available in the online journal.)

Monte-Carlo scheme, all light travel time effects (LTTE) are considered.

\subsection{D Multi-Zone Synchrotron Polarization (3DPol) Code}

We employ a similar geometry setup in the 3D multi-zone synchrotron polarization code. As a full 3D description is necessary to evaluate the polarization, we further divide the emission region evenly in the $\phi$ direction (Figure 1). The assumption of axisymmetry of all parameters is still kept. As in the MCFP code, all calculations are performed in the comoving frame of the emission region. The LOS is properly transformed via relativistic aberration. In each zone, we project the magnetic field onto the plane of sky in the comoving frame, and use the time-dependent electron distribution generated by the MCFP simulation, instead of a simple power-law, to evaluate the polarization via (Rybicki \& Lightman 1985)

$$
\Pi(\omega)=\frac{P_{\perp}(\omega)-P_{\|}(\omega)}{P_{\perp}(\omega)+P_{\|}(\omega)},
$$

where $\omega$ is the frequency, $P_{\|}(\omega)$ and $P_{\perp}(\omega)$ are the radiative powers with electric-field vectors parallel and perpendicular to the projection of the magnetic field onto the plane of the sky, respectively. $P_{\|}(\omega)$ and $P_{\perp}(\omega)$ are obtained via integration of the single-particle powers $P_{\|}(\omega, \gamma)$ and $P_{\perp}(\omega, \gamma)$ over the electron spectrum $N_{e}(\gamma)$, e.g., $P_{\|}(\omega)=\int_{1}^{\infty} P_{\|}(\omega, \gamma) N_{e}(\gamma) d \gamma$. Our treatment of synchrotron polarization is restricted to the optically thin regime, since we focus on high-frequency radio through optical/UV polarization. Since the net electric-field vector is perpendicular to the projected magnetic field on the plane of sky in the comoving frame, the electric vector position angle, also known as polarization angle (P.A.), is obtained for each zone, hence we can obtain the Stokes parameters (without normalization) at every time step via

$$
(I, Q, U)=L_{\nu} *\left(1, \Pi \cos 2 \theta_{E}, \Pi \sin 2 \theta_{E}\right),
$$

where $L_{v}$ is the spectral luminosity at frequency $\nu, \Pi$ is the polarization percentage and $\theta_{E}$ is the electric vector position angle for that zone. The code then calculates the relative time delay to the observer for each zone, so as to take full account of the external LTTE, i.e., the time delay in the observed emission due to the spatial difference of each zone in the emission region. The internal LTTE, which is introduced through Compton scattering, is irrelevant for the present discussion of synchrotron polarization. Since the emission from different zones is incoherent, the total Stokes parameters are then calculated by direct addition of the Stokes parameters for each zone from which emission arrives at the observer at the same time. In a post-processing routine to analyze the 3DPol output, we normalize the total Stokes parameters at every time step to evaluate the polarization, and transform the result back to the observer's frame.

In order to test our code, we compare the total, time-dependent synchrotron spectra obtained with our 3DPol code, with the result of the MCFP code. This comparison is illustrated in Figure 2, which shows the SEDs of the synchrotron hump and light curves obtained by the MCFP and 3DPol codes when applied to reproduce the observed variability in Mkn 421 considered by Chen et al. (2011) in the framework of flaring scenario 4, i.e., when the effect of the shock is to inject additional non-thermal electrons into the shocked region (these MCFP results are considered in more detail in Section 3.4). The overall agreement is excellent, although minor differences can be noticed, which can be attributed to the fact that the two codes use different ways to treat the radiation transfer. In particular, the larger number of zones in the 3D geometry used within 3DPol leads to slightly different LTTE compared to those in the 2D geometry of the MCFP code.

\section{RESULTS}

In this section, we present case studies for two blazars as examples to apply our polarization code: the high-frequencypeaked BL Lac object (HBL) Mkn 421 and the Flat-Spectrum Radio Quasar (FSRQ) PKS 1510-089. Mkn 421 exhibited a correlated X-ray and $\gamma$-ray flare in 2011 March (Fossati et al. 2008), which was monitored with excellent X-ray and $\gamma$-ray coverage throughout the entire flare. PKS 1510-089 exhibited extended flaring activity in 2008-2009. A particular flare in 2009 March was well covered by monitoring observations in the infrared, optical, X-rays, and $\gamma$-rays (Abdo et al. 2010a; D'Ammando et al. 2011; Marscher et al. 2010). Chen et al. $(2011,2012)$ presented model fits to snap-shot SEDs and light curves of these two blazars, using their shock-in-jet model 
as described above. For Mkn 421, Chen et al. (2011) successfully modeled both snap-shot SEDs and light curves with a pure synchrotron-self-Compton (SSC) model. For PKS 1510-089, Chen et al. (2012) found that, for the 2009 March flare, both a pure SSC model and an SSC+EC model produced reasonable but imperfect fits. A pure SSC model predicted too hard spectral slopes at X-ray and infrared frequencies, while an EC model achieved satisfactory fits to snap-shot SEDs and light curves, at the expense of an extremely short particle escape timescale, which awaits further explanation. In the most favorable EC model by Chen et al. (2012), the external radiation field was the infrared radiation from a dusty torus.

In our polarization variability study, we will use similar parameters as in Chen et al. $(2011,2012)$ and compare the polarization signatures from all potential flaring scenarios 1-4, as described above. In order to facilitate a direct comparison, we choose the same initial parameters for all scenarios. The parameters are chosen in a way that they produce adequate flares for both sources in order to allow for direct comparisons and to mimic the observational data. Since both MCFP and $3 \mathrm{DPol}$ codes are time-dependent, in the beginning there is a period for the electrons and the photons to reach equilibrium, before we introduce the parameter disturbance produced by the shock. The light curves shown in all our plots start after this equilibrium has been reached. As the flaring activity in the four scenarios exhibits different characteristics in duration and in strength, we define similar phases in the flare development for the purpose of a direct comparison. These phases correspond approximately to the early flare, flare peak, and late flare, and post-flare (end) states. As in Chen et al. (2011, 2012), the ratio between the emission-region dimensions $z$ and $r$ is chosen to be $4 / 3$, to mimic a spherical volume.

Due to the relativistic aberration, even though we are observing blazars nearly along the jet in the observer's frame (typically, $\theta_{\mathrm{obs}}^{*} \sim 1 / \Gamma$, where $\Gamma$ is the bulk Lorentz factor of the outflow along the jet), the angle $\theta_{\text {obs }}$ between LOS and the jet axis in the comoving frame it is likely much larger. Specifically, if $\theta_{\mathrm{obs}}^{*}=1 / \Gamma$, then $\theta_{\mathrm{obs}}=\pi / 2$. Hence, for our base parameter studies, we set $\theta_{\text {obs }}=90^{\circ}$. This choice turns out to have a considerable effect on the result, which will be discussed in Section 4.1.

We define the P.A. in our simulations as follows. P.A. $=0^{\circ}$ corresponds to the electric-field vector being parallel to the projection of the jet on the plane of sky. Increasing P.A. corresponds to counter-clockwise rotation with respect to the LOS, to $180^{\circ}$ when it is anti-parallel to the projected jet (which is equivalent to $0^{\circ}$ due to the $180^{\circ}$ ambiguity). In all runs the zone numbers in three directions are set to $n_{z}=30, n_{r}=27$ and $n_{\phi}=120$, which we find to provide appropriate resolution. As is mentioned in Section 1, we will only focus on a parameter study and compare the general flux and polarization features of each scenario. All results are shown in the observer's frame.

Table 1 lists some key parameters. We assume that the initial nonthermal electron density $n_{e}$ is the same in every zone. The initial electron spectrum has a power-law shape with index $p$ and minimum and maximum cutoff energies $\gamma_{\min }$ and $\gamma_{\max }$, which will evolve according to the Fokker-Planck equation. The entire emission region is a cylinder of a length $Z$ and a radius $R$, with a bulk Lorentz factor $\Gamma$, while the stationary shock is also a cylinder of radius $R$ but of length $z=Z / 10$. The helical magnetic field has a magnitude $B$ and pitch angle $\theta_{B}$. In all cases that we discuss in this paper, the initial magnetic field is oriented at $\theta_{B}=45^{\circ}$, so that the toroidal and the poloidal
Table 1

Summary of Model Parameters

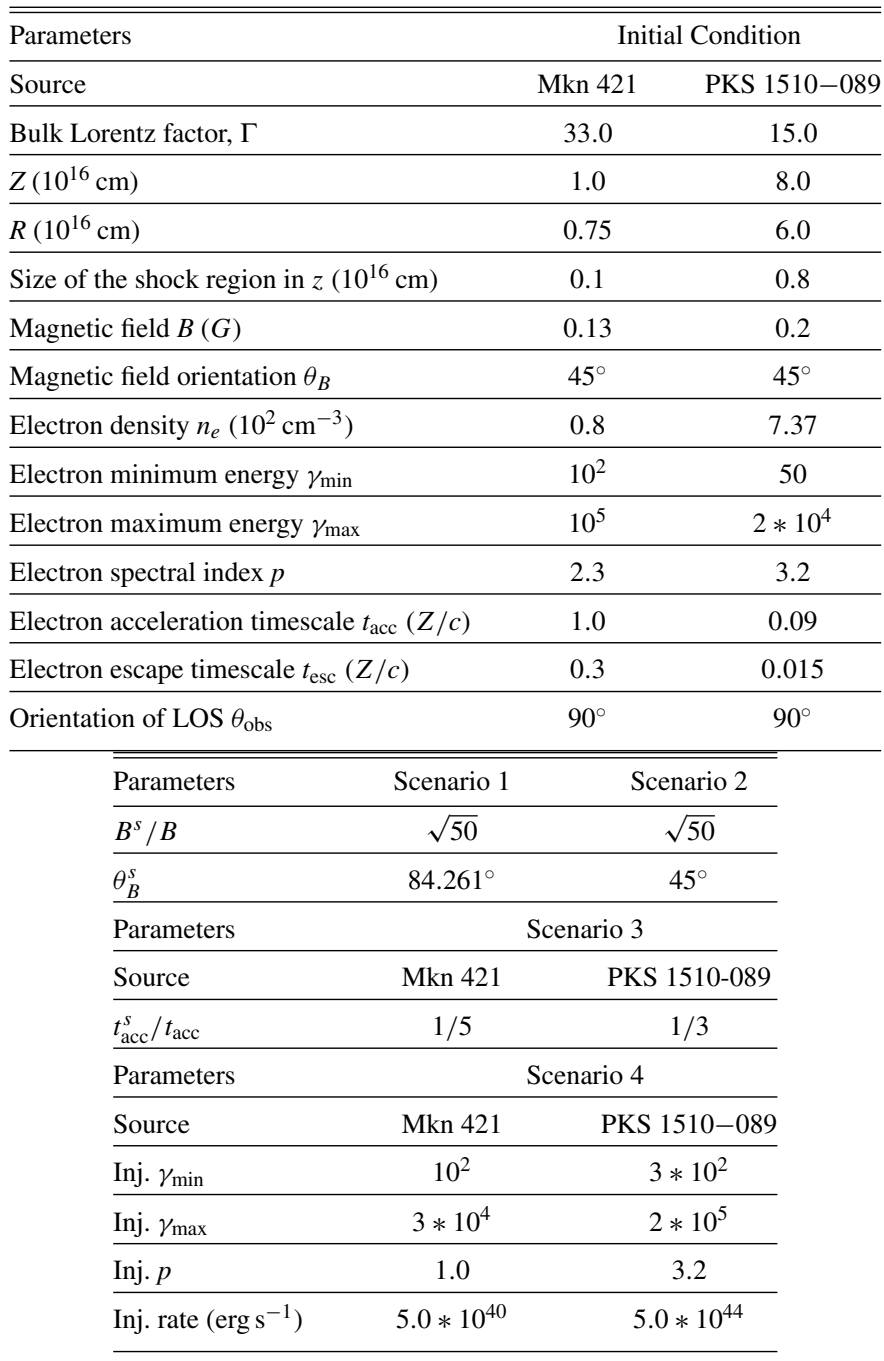

Notes. Top: initial parameters. Notice that $\gamma_{\min }, \gamma_{\max }$ and $p$ can change before the electrons reach pre-flare equilibrium. Bottom: shock parameters for each scenario. s-superscript indicates the parameters during the shock. For scenarios 1 and 2 , we chose the same shock parameters for both sources. The parameter $B_{s} / B$ is the magnetic-field amplification factor. $\theta_{\mathrm{B}}^{s}$ is the magnetic-field pitch angle in the shocked region. For Scenario 4, we list the parameters for the injected electron distribution in the shocked region.

components of the magnetic field are equal. This choice is not required, but it aids to illustrate that even with equal toroidal and poloidal components, the polarization will have an excess from the poloidal component of the magnetic field, due to the fact that the projection of the two components onto the plane of sky will generally not be equal, as we will show in the following.

\subsection{Change of the Magnetic Field Orientation}

In this scenario, the shock instantaneously increases the toroidal magnetic-field component at its location, so as to increase the total magnetic field strength and change its orientation in those zones. The new magnetic field will be kept until the shock moves out of the zone; at that time, it reverts back to its original (quiescent) strength and orientation due to dissipation.

For Mkn 421, since both synchrotron and SSC are proportional to the magnetic field strength, we see flares in both 

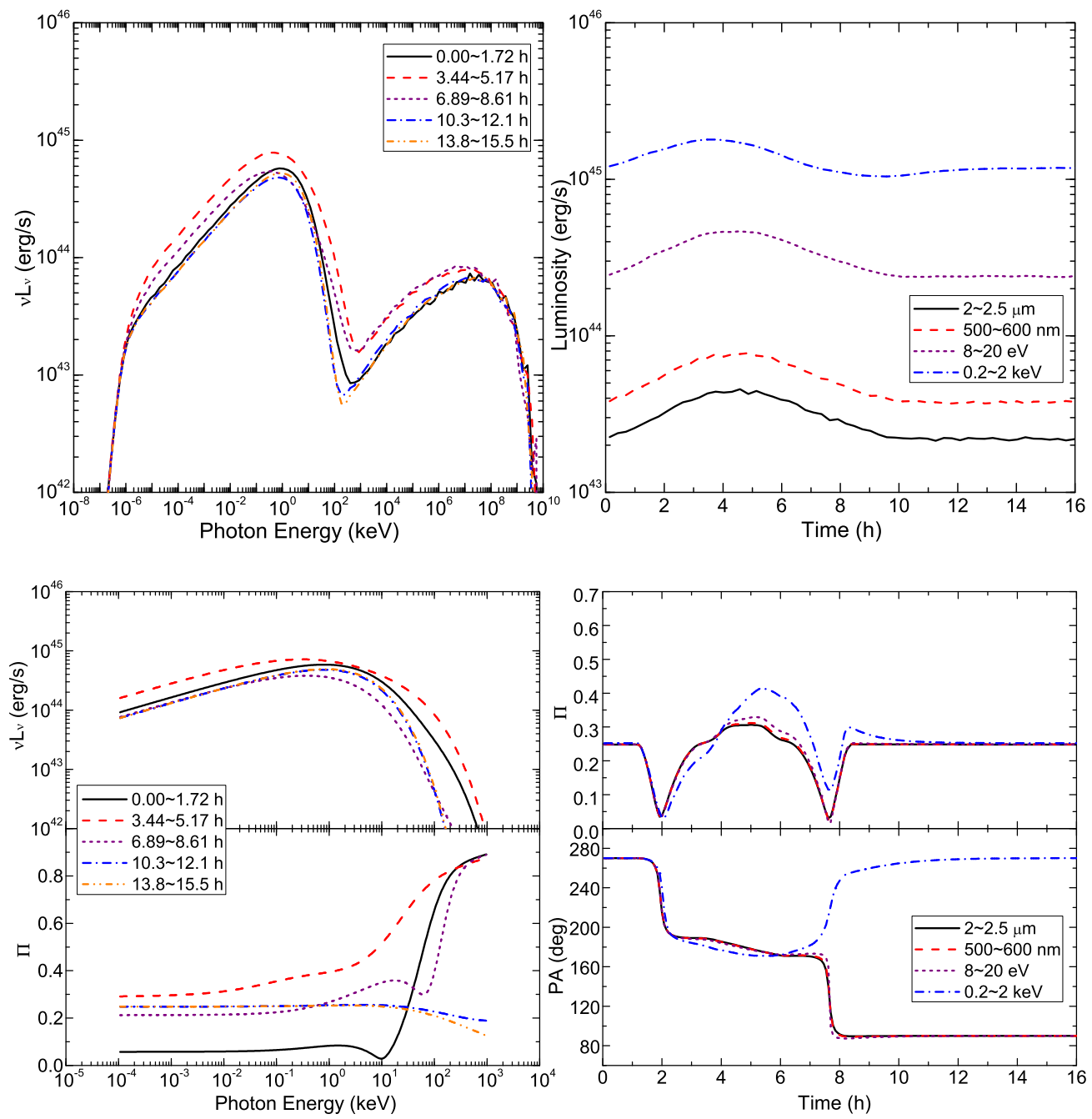

Figure 3. Flaring scenario 1 (change of direction and strength of the magnetic field) for Mkn 421. Upper left: the SEDs of Mkn 421 from the MCFP code. SEDs are chosen at approximately the beginning of the flare (black solid), peak (red dash), after peak (purple short dash), ending (blue dash dot) and back to equilibrium (orange dash dot dot), with the same time bin size. Upper right: the light curves of Mkn 421 from the MCFP code, chosen at infrared (black solid), optical $V$ band (red dash), UV (purple short dash) and soft X-ray (blue dash dot) frequencies. Lower left: the synchrotron SEDs (top) of Mkn 421 from the 3DPol code, and the polarization percentage vs. photon energy (bottom). The time bins are chosen the same as SEDs from MCFP. Lower right: The polarization percentage vs. time (top), and the polarization position angle vs. time (bottom). The energy bands are chosen the same as the light curves from MCFP.

(A color version of this figure is available in the online journal.)

spectral bumps, though the $\gamma$-ray flare has a much lower amplitude (Figure 3, upper left). It is also obvious that the polarization percentage has a dependence on the photon energy, although patterns above $\sim 10 \mathrm{keV}$ are resulting from the electron distribution cutoff. In addition, it also has a time dependency. We notice that the synchrotron emission from the unshocked jet has a polarization percentage of about $25 \%$. This is because the projected poloidal component $B_{z}$ on the plane of sky is larger than the projected toroidal component $B_{y}$, so that there is an excess in the contribution of the poloidal component to the polarization. The value of 0.25 is specific to the choice of initial conditions with $\theta_{B}=45^{\circ}$, and would change for different values of $\theta_{B}$ and $\theta_{\text {obs }}$ and/or electron spectral index. As will be discussed in detail below, this geometric effect plays a significant role for the origin of polarization signatures. It is interesting to note that, unlike the light curve, which is symmetric in time, the polarization percentage has an asymmetric time profile, especially for higher energies. Furthermore, the polarization angles are shown to have $\sim 180^{\circ}$ swings, although the $\mathrm{X}$-ray polarization angle reverts back to its original orientation after the initial $\sim 90^{\circ}$ rotation, instead of continuing to rotate in the same direction, as in the lower-frequency bands. As we will elaborate in detail below, all these phenomena can be explained as the combined effect of electron evolution and LTTE.

Since we assume that every zone in the jet has identical initial conditions and the shock will affect the same change everywhere, we can simply choose one zone to represent the electron evolution of the emission region (strictly speaking, due to internal LTTE and other geometric effects, different zones will be subject to slightly different SSC cooling rates; however, we have carefully checked the electron spectra and found this effect to be negligible in the cases studied here). This is illustrated in Figure 4 (left). When the shock reaches the zone, it will increase the magnetic field strength, hence synchrotron cooling becomes faster. Therefore the electron spectrum becomes softer while the shock is present, especially at higher electron energies, resulting in a higher possible maximal polarization percentage $\left(\Pi_{\max }=(p+1) /(p+7 / 3)\right.$, where $p$ is the local electron spectral index in the energy range responsible for the synchrotron emission at a given frequency; see Rybicki \& Lightman (1985); hereafter $\Pi_{\max }$ stands for the theoretical maximal polarization percentage for a given nonthermal electron spectrum in one 


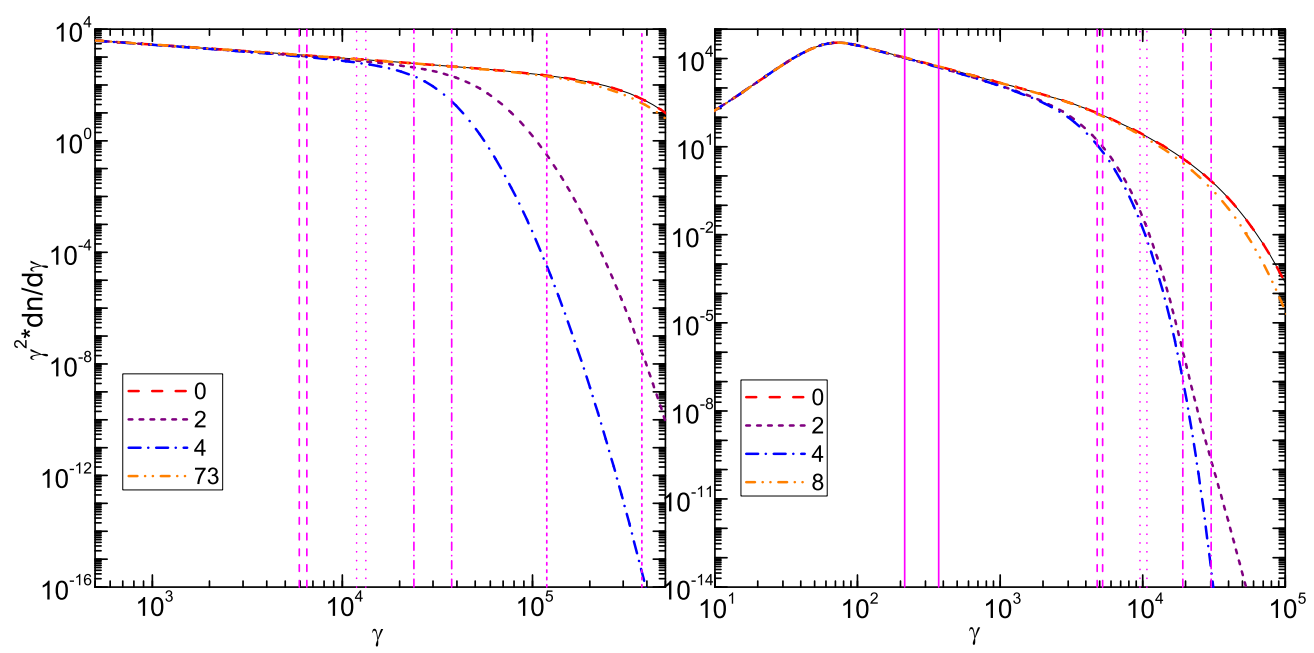

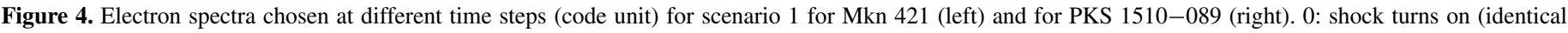

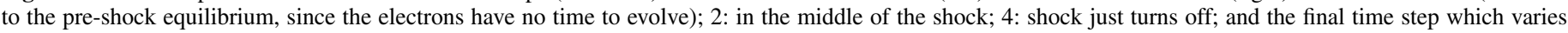

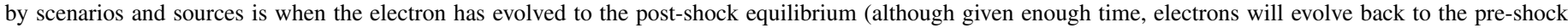

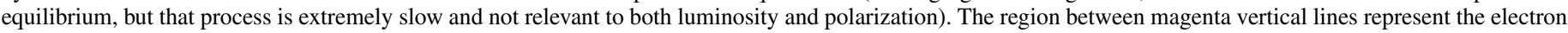

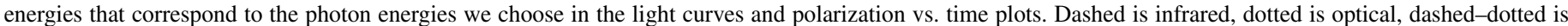
$\mathrm{UV}$, solid is radio (PKS 1510-089 only), and short-dashed is X-rays (Mkn 421 only).

(A color version of this figure is available in the online journal.)

zone, irrespective of any contaminations or LTTEs). After the shock leaves the zone, the electrons gradually evolve back to equilibrium. This process takes longer at higher energies, hence the polarization percentage for more energetic photons recovers more slowly. Nevertheless, the X-ray light curve appears to evolve faster than at the lower-frequency ones. The reason for this is that the flare amplitude (compared to the equilibrium emission) is so low at X-ray frequencies, that even if the electrons have not yet reached equilibrium near the end of the flare, their contribution to the total synchrotron flux is negligible.

We now discuss the influence of LTTEs on the polarization signatures. The situation is illustrated in Figure 5. In equilibrium, the Stokes parameter $U$ will cancel out because of the axisymmetry. Additionally, despite the fact that $\theta_{B}=45^{\circ} \mathrm{im}-$ plies $B_{\phi}=B_{z}$, the "effective toroidal component," $B_{y_{0}}$, i.e., the $y_{0}$ component of $B_{\perp}$ on the plane of sky in the comoving frame, which is generally a fraction of $B_{\phi}$, is lower than the "effective poloidal component," which is equal to $B_{z}$. Thus, the polarization is dominated by $B_{z}$. Therefore, at the initial state, the polarization percentage is relatively low, and the polarization position angle is at $270^{\circ}$ (or $90^{\circ}$, considering the $180^{\circ}$ ambiguity). However, when the shock reaches the emission region, $B_{\phi}$ begins to dominate. Due to LTTE, the observer will initially only see the $+x$ side of the flaring region (Figure 5, left), which has a preferential magnetic field predominantly in the $+y_{0}$ direction (Figure 5, left and Figure 6, red). Since the emission from the flaring region is much stronger than other parts of the emission blob, it will quickly cancel and then dominate over the polarization caused by $B_{z}$ in the background region. Hence, the polarization percentage will first drop to almost zero and then rapidly increase, while the P.A. will drop to $\sim 180^{\circ}$, representing an electric-field vector directed along the jet, caused by the dominant $B_{y_{0}}$. Furthermore, the electron spectrum evolves relatively slowly and the cooled high-energy electrons give rise to very high $\Pi_{\max }$. Therefore, the observed polarization will have contributions not only from the flaring region, but also from zones with more evolved electron distributions, where the shock has passed recently. We can observe in Figure 5 that this "polarization region" is not symmetric from pre-peak to postpeak, resulting in an asymmetry in time, especially at higher energies.

There is, however, one additional factor. We can see in the light curves (Figure 3, upper right) that the X-ray flareto-equilibrium ratio is much smaller than at lower energies. Hence, in X-rays, the flaring region takes longer to dominate the polarization patterns, and they revert back to equilibrium faster. Furthermore, high energy electrons take longer to evolve back to equilibrium. This is because our MCFP code treats the acceleration timescale $t_{\text {acc }}$ as energy-independent. Therefore, since the high-energy end of the electron spectrum has been entirely depleted of electrons during the flaring event, the highest electron energies will be the last to be gradually re-populated from lower energies, while still providing a considerable $\Pi_{\max }$. For this reason, the X-ray polarization region will be much larger than at lower energies. Therefore, when the flaring region moves to $-x$ in Figure 5, where the preferential magnetic field is directed in $-y_{0}$, the X-ray photons will be dominated by the evolving and the background region on the $+x$ side, although lower energy photons are still dominated by the flaring region. This gives rise to the interesting phenomenon that at lower energies the P.A. will continuously drop to $90^{\circ}$ (which is equivalent to $270^{\circ}$ because of the $180^{\circ}$ ambiguity) as the polarization region gradually moves to $-x$ and out of the emission region; while for X-rays it instead reverts back, as the evolving region on the $+x$ side dominates the polarization, causing the magnetic field again to be preferentially oriented in the $+y_{0}$ direction, mimicking the pre-peak situation.

PKS 1510-089 presents a similar situation, although there are some major differences. First, PKS1510-089 requires a dominating EC component at $\gamma$-ray energies, which is independent of the magnetic field strength. Thus in the current scenario, no flare is visible in the Compton bump (Figure 7, upper left). Also, due to the contamination of the external thermal radiation from the dust torus in the optical and UV bands (Figure 7, left), which is unpolarized, the observed polarization percentage 

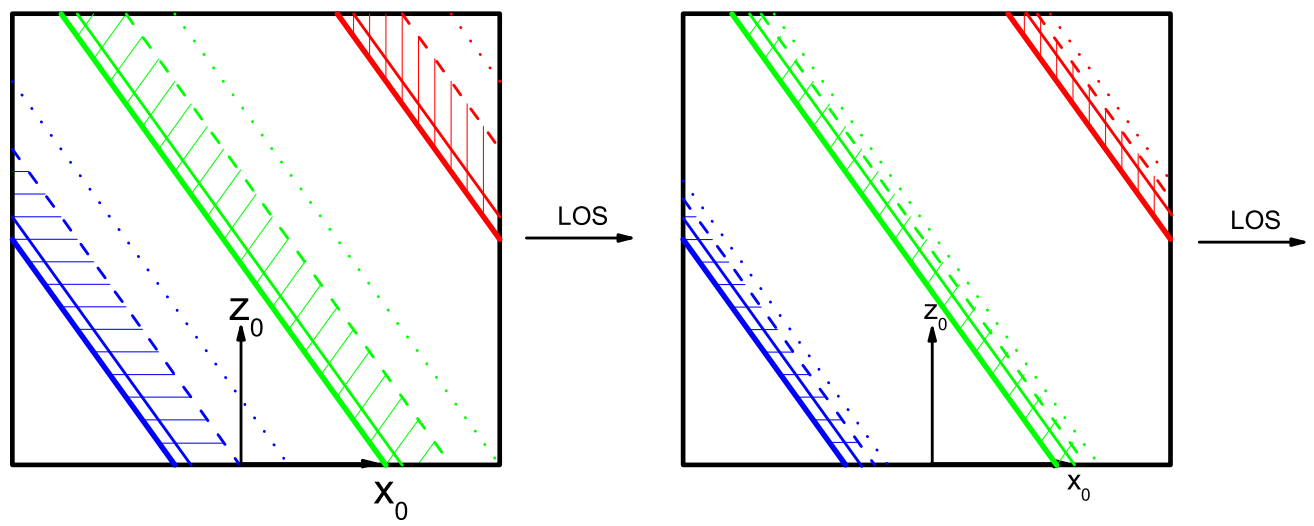

Figure 5. Illustrations of a vertical slice of the cylindrical emission region at different times. Left: illustration for Mkn 421. Right: illustration for PKS 1510-089. The LOS is assumed to be directed in $+x$. The shock propagates through the jet from top to bottom. Solid lines demarcate regions in the jet where the shock is present at equal photon-arrival times at the observer. Red (approximately red in Figure 1) corresponds to the pre-peak observer time, green (approximately cyan and yellow in Figure 1) represents the flare peak and blue (approximately blue in Figure 1) the post-peak time. The flaring region is between the bold and the thin solid lines, which correspond to the zones where the shock is currently present. The shaded region between the bold solid line and the dashed line is what we call the polarization region, containing the flaring region and the evolving region of recently shock-accelerated electrons. The dotted line represents the zones where electrons have evolved to the post-flare equilibrium. Although the electrons in the region between the dashed line and the dotted line are still evolving, their contribution to the polarization is negligible. Hence all points outside the polarization region are called the background region, or the nonflaring region.

(A color version of this figure is available in the online journal.)

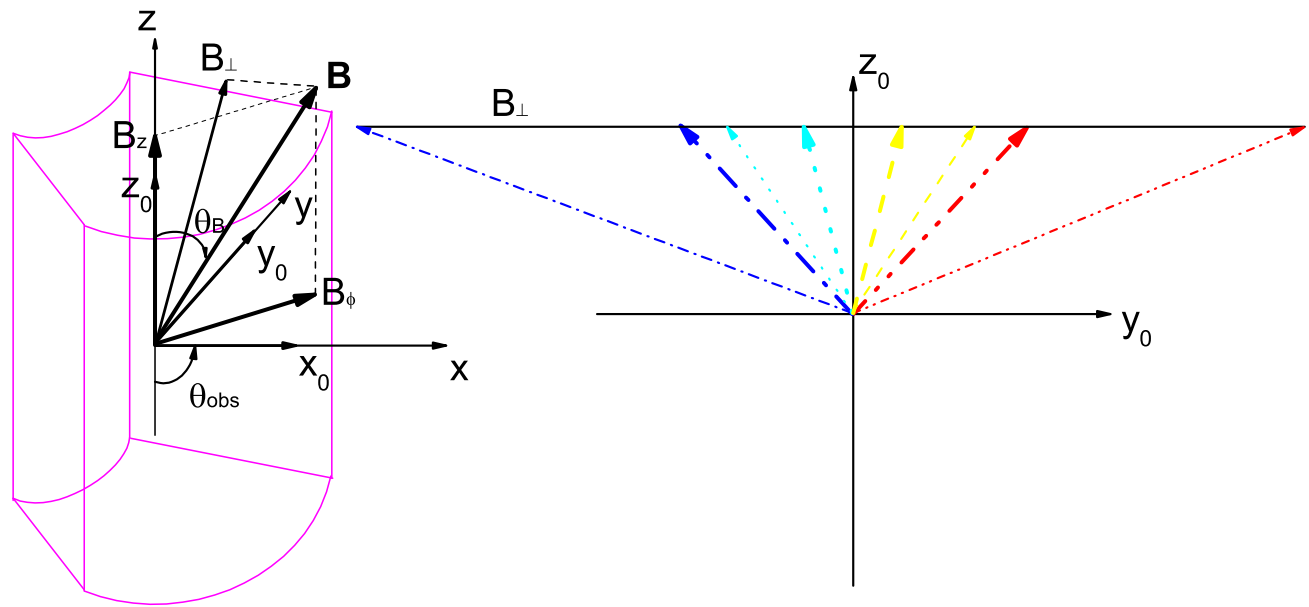

Figure 6. Illustrations of magnetic field in the emission blob for $\theta_{\mathrm{obs}}=90^{\circ}$. Left: illustration for the magnetic field in the magenta zone shown in Figure 1. The coordinates are illustrated in Figure 1. The total magnetic field $\mathbf{B}$ in that zone is assumed to have only two components, $B_{z}$ and $B_{\phi} ; \theta_{B}$ is the angle between $\mathbf{B}$ and the $z$-axis. $B_{\perp}$ is the projection of $\mathbf{B}$ on the plane of sky $\left(y_{0}, z_{0}\right)$. Right: illustration for $B_{\perp}$ at different locations in the emission region. Red, yellow, cyan and blue correspond to the regions shown in Figure 1. When $\theta_{\mathrm{obs}}=90^{\circ}$, the $z_{0}$ component of $B_{\perp}, B_{z_{0}}=B_{z}$ throughout the emission blob, while $B_{y_{0}} \leqslant B_{\phi}$, and equality is obtained at the $x=+r_{\max }$ (red) and $x=-r_{\max }$ (blue) boundaries. The bold arrows represent the initial magnetic field orientation $\left(\theta_{B}=45^{\circ}\right)$, while the narrow arrows illustrate the magnetic field orientation change in scenario $1\left(\theta_{B} \sim 84^{\circ} .3\right)$.

(A color version of this figure is available in the online journal.)

will be considerably diminished, especially at UV wavelengths. On the other hand, the EC fit required softer electron spectra (Figure 4, right); consequently, $\Pi_{\max }$ (the maximum possible polarization in the non-thermal synchrotron component) is much higher. Therefore, at the flare peak, the polarization percentage rises up more than that in Mkn 421. Additionally, the relative electron evolution rate is faster (although the total flare time is longer than that of Mkn 421, due to the larger dimensions of the emission region). As a result, the polarization region is much smaller, nearly equivalent to the flaring region (Figure 5, right). This will make the polarization region highly symmetric from pre-peak $(+x)$ to post-peak $(-x)$, so that the time asymmetry found in Mkn 421 is not present in the case of PKS 1510-089. Additionally, at the flare peak the polarization region itself will concentrate on the central region (green) in Figure 5, where $B_{y_{0}}$ is much weaker. Thus, the polarization dominated by the effective toroidal component will be diminished to a certain extent, creating a plateau at the flare peak, which is much lower than $\Pi_{\max } \simeq 0.75$. In fact, we also find a similar but weaker effect in Mkn 421, due to a larger polarization region; at X-rays, however, the very large polarization region suppresses this effect, which is why the X-ray polarization percentage exhibits a pronounced peak.

Comparing the predicted P.A. swings in Figures 3 and 7 (lower right) to the ones observed in several blazars, in connection with $\gamma$-ray flaring activity, one notices that the observed P.A. swings are more gradual than the ones predicted here. However, we remind the reader that we employ the simple assumption that the magnetic field is instantaneously changed by the shock. In reality, this change might occur over a finite amount of time. While we have not investigated such a scenario in our simulations, one might suspect that, with a more realistic time profile of the magnetic-field change, the predicted P.A. swing will be much smoother, instead of two rapid drops and a plateau 

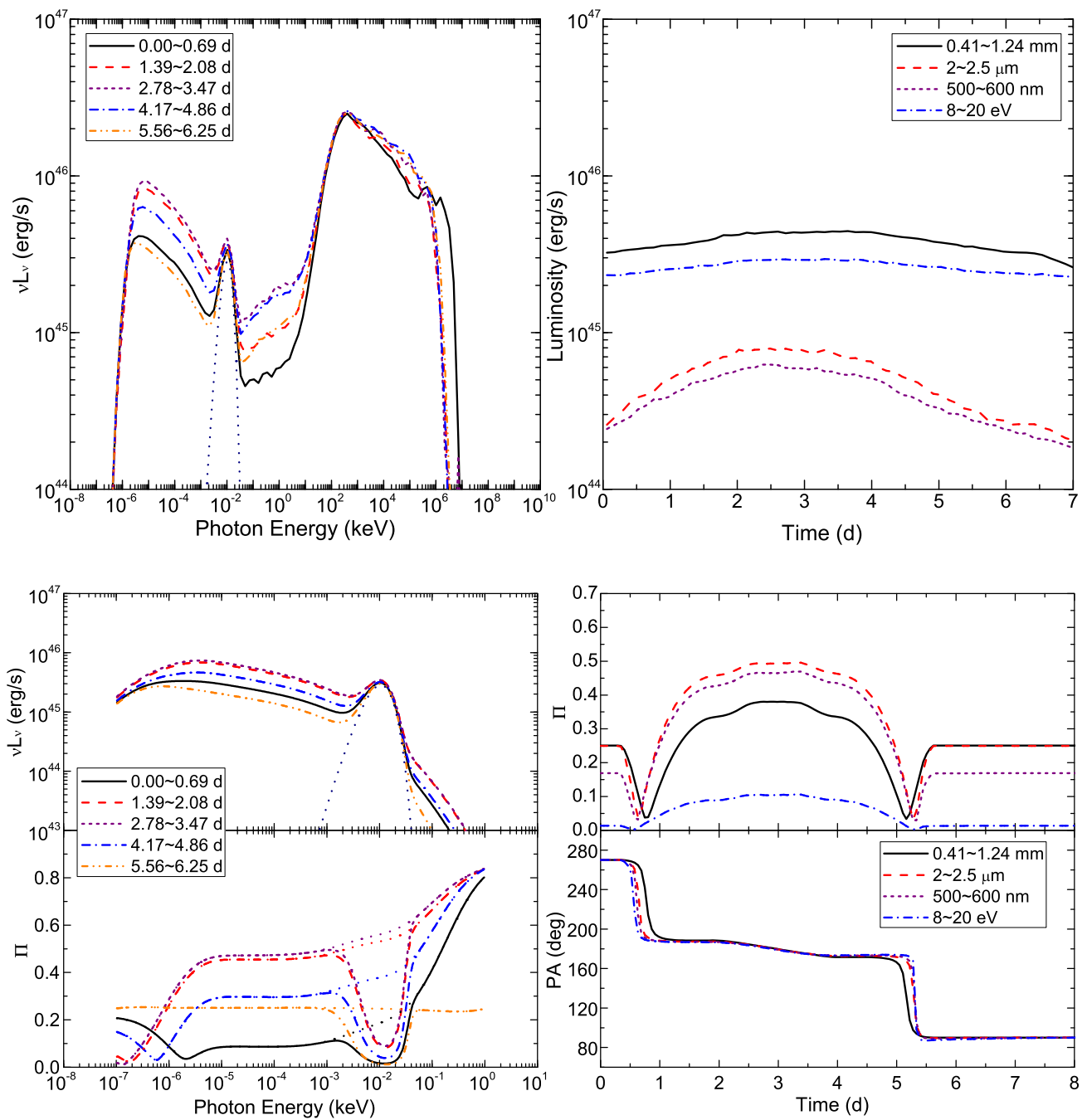

Figure 7. Flaring scenario 1 (change of direction and strength of the magnetic field) for PKS 1510-089. Upper left: SEDs of PKS 1510-089 including the external photon field contribution, at approximately the beginning of the flare (black solid), before peak (red dashed), peak (purple short-dashed), after peak (blue dash-dotted) and back to equilibrium (orange dashed dot-dotted), with the dotted line for the external photon field contribution. Upper right: the light curves including the external photon field contribution, at radio (black solid), infrared (red dashed), optical $V$ band (purple short-dashed), UV (blue dash-dotted). Lower left: the synchrotron SEDs, including the external photon field, from 3DPol (top), with the dotted line for the external photon field contribution; and the polarization percentage vs. photon energy with the external photon contamination considered (bottom), where dotted lines represent the polarization percentage without the contamination. Lower right: the polarization percentage vs. time with external contamination (top), and P.A. vs. time (bottom).

(A color version of this figure is available in the online journal.)

in between. Note also that rather step-like P.A. rotations, similar to the features found in our simulations, have in fact been observed in S5 0716+714 by Ikejiri et al. (2011). We also point out that the $\sim 180^{\circ}$ P.A. swing we show here is the result of one individual disturbance moving through the jet. If there are multiple disturbances (flares) in succession, the P.A. will rotate up to $180^{\circ}$ times the number of flares.

There is an ambiguity in the helical magnetic field handedness. In our model setup, we chose it to be right-handed and against the bulk motion direction. If it were left-handed, the P.A. rotation would appear to be in the opposite direction, but everything else would remain the same. Also notice that even the light curves will not be symmetric in time because of the asymmetry in time between the dynamics of the shock moving through the emission region and the electron cooling. However, in cases where the size of the active region, energized by the passing shock, is much smaller than the overall jet emission region (e.g., due to dominant EC cooling, the timescale for electron evolution in PKS 1510-089 is much shorter than in the case of Mkn 421), this effect is minor, yielding nearly symmetric light curves.

The $\gamma$-ray emission from PKS $1510-089$ is due to EC, for which changes in the synchrotron component are irrelevant, while the light curve features in our code are identical to those resulting from the MCFP code, as presented in Chen et al. (2011, 2012). In the discussion of the following scenarios, we will show the time-dependent SEDs and light curves only for Mkn 421, and restrict the discussion of PKS 1510-089 to the polarization signatures.

\subsection{Increase of the Magnetic Field Strength}

In this scenario, we assume that the shock only increases the total magnetic field strength at its location, leaving its orientation unchanged. Since the electron evolution is independent of the magnetic field orientation, it appears identical to the above scenario (Figure 4). The same applies to the SEDs and light curves. However, the polarization patterns in time show major 

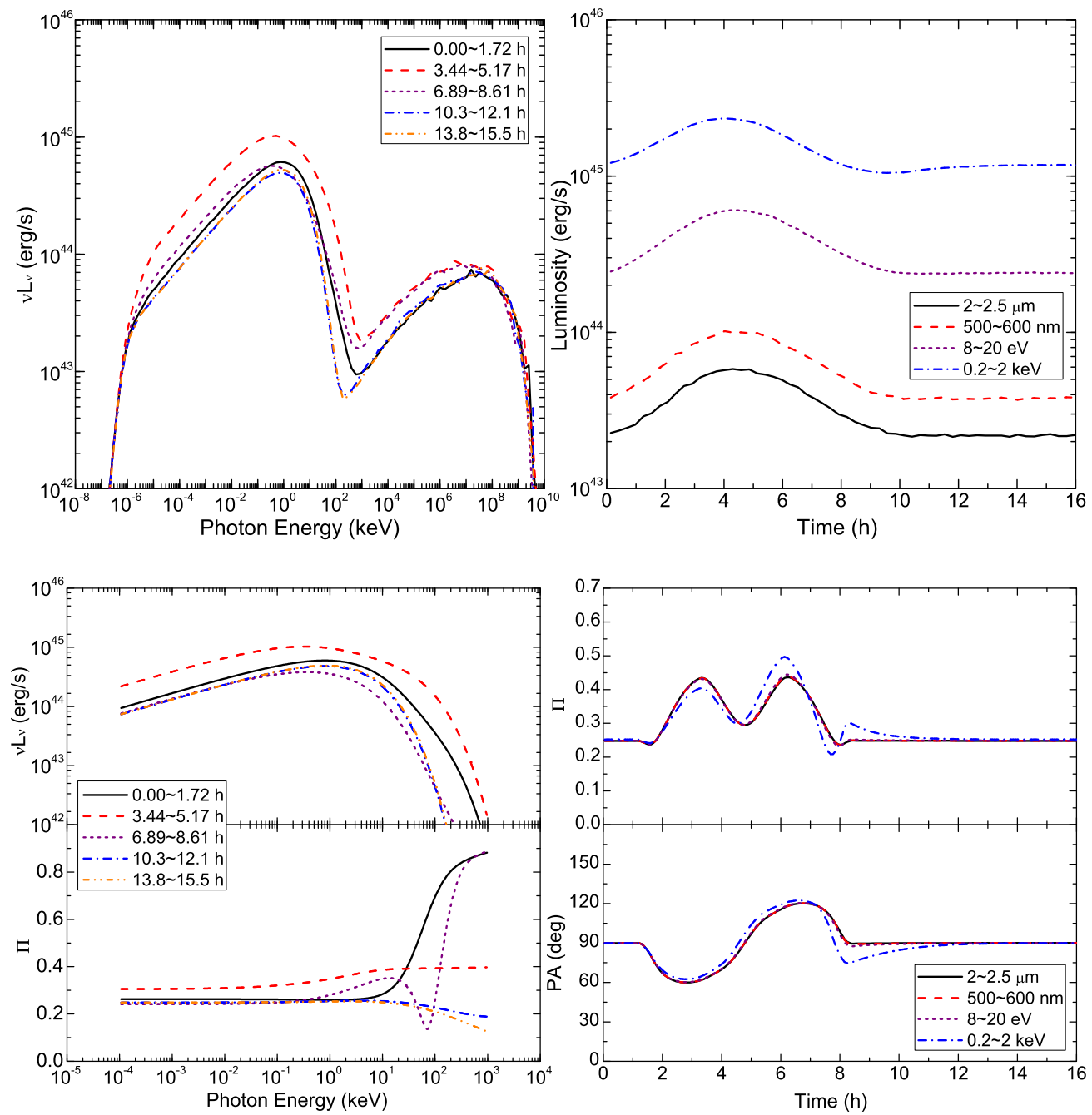

Figure 8. Flaring scenario 2 (increasing magnetic-field strength with unchanged orientation) for Mkn 421. Panels and line styles are as in Figure 3.

(A color version of this figure is available in the online journal.)
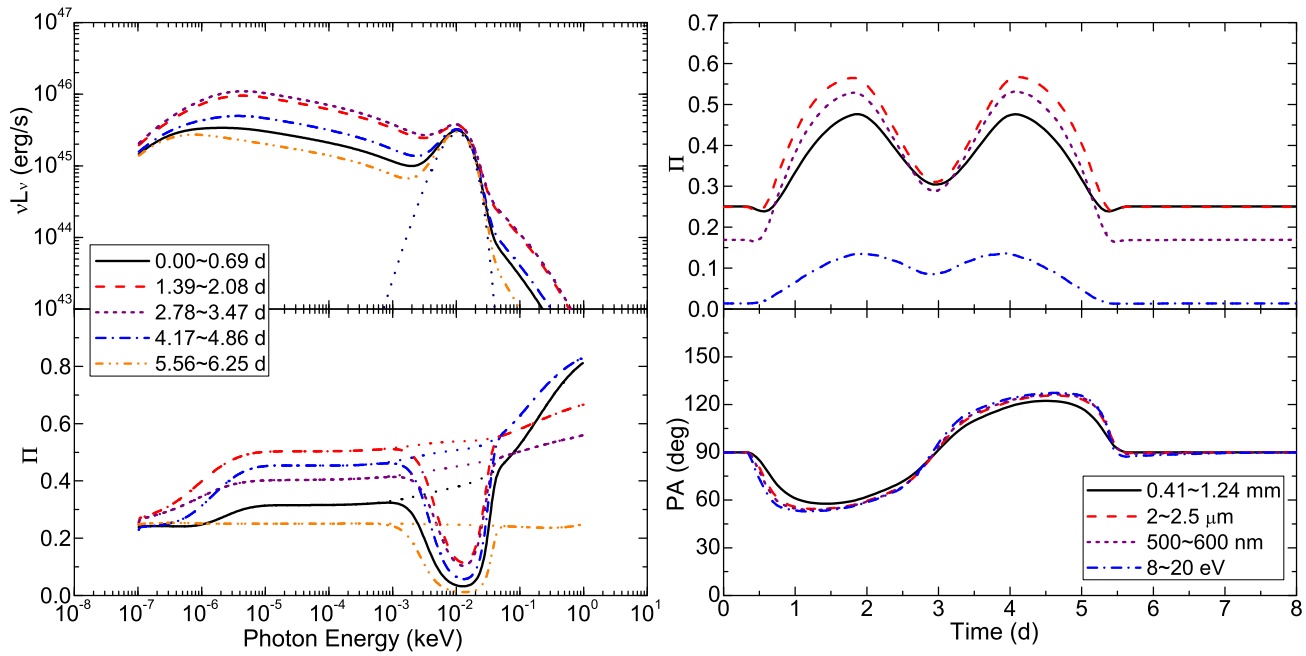

Figure 9. Flaring scenario 2 (increasing magnetic-field strength with unchanged orientation) for PKS 1510-089. Panels and line styles are as in the bottom two panels of Figure 7.

(A color version of this figure is available in the online journal.)

differences (see Figure 8 for Mrk 421 and Figure 9 for PKS 1510-089). Since the magnetic field is oriented at $45^{\circ}$ to the $z$-axis, $B_{\phi}$ and $B_{z}$ will be equal throughout the emission region. Due to axisymmetry, $B_{\perp}$ in the polarization region will be confined in a cone of $\pm 45^{\circ}$. Therefore, the polarization induced by $B_{z}$, is always dominant, so that the P.A. will be confined to at most $\left(45^{\circ}, 135^{\circ}\right)$. Since also the polarization of the background region is dominated by the effective poloidal 

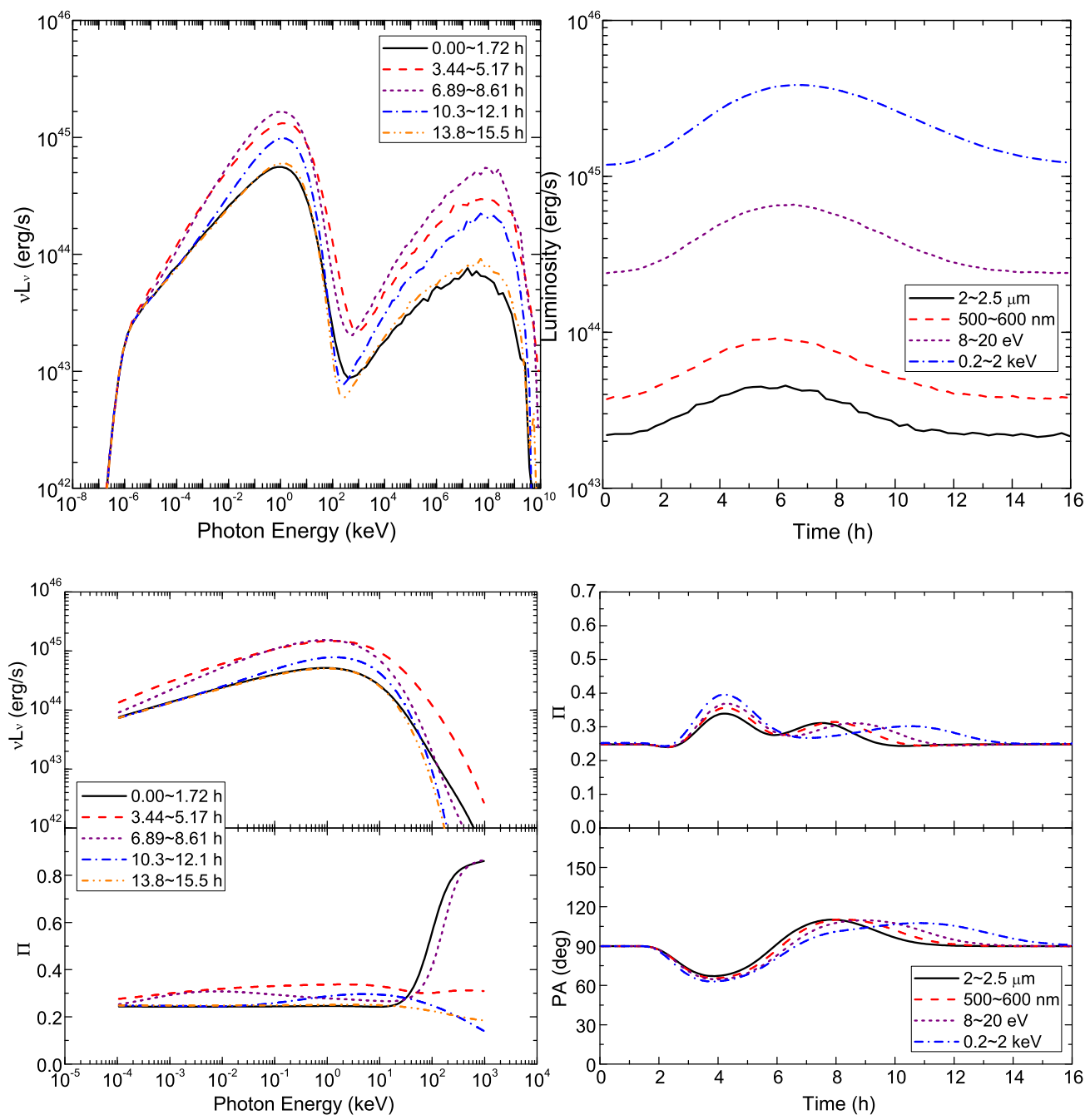

Figure 10. Scenario 3 (shortened acceleration timescale) for MKN 421. Panels and line styles are as in Figure 3.

(A color version of this figure is available in the online journal.)

component, these two will add up, resulting in a slightly higher maximal polarization percentage compared to scenario 1. However, in the immediate neighborhood of the starting and ending points of the flare, the situation is a little bit different: although the effective toroidal component $B_{y_{0}}$ is still weaker than $B_{z}$, the two components are closer in magnitude. Hence $B_{\phi}$ will diminish the polarization percentage by a small amount. Thus the two sharp dips shown in the previous polarization percentage patterns become much smaller.

After that, again due to LTTE, only the $+x$ side of the flaring region is observed initially, which has a preferential magnetic field oriented at $\sim-135^{\circ}$ (Figure 6). Therefore, the polarization is dominated by Stokes parameter $U$. Hence we observe that the polarization percentage increases and the P.A. moves to $45^{\circ}$. However, a basin forms at the flare peak, replacing the previous plateau, and the P.A. moves back to $90^{\circ}$. This is because the polarization region at the flare peak (green in Figures 5 and 6) is dominated by $B_{z}$, while the background region on the $-x$ and $+x$ side is just like the initial state. Therefore, the Stokes parameter $U$ contributions will cancel out due to axisymmetry, leaving the polarization dominated by $B_{z}$. The same applies when the polarization region moves to the post-peak position (blue in Figure 5). Slight differences in the X-ray behavior are again explained by the slower electron evolution back to equilibrium.

\subsection{Shortening of the Acceleration Timescale}

In this scenario, the shock is assumed to lead to more efficient particle acceleration by instantaneously shortening the local acceleration timescale. As a result, the electrons will be accelerated to higher energy, leading to flares in both synchrotron and Compton emission (Figures 10, upper left, 11 left). At the same time, the peaks of both spectral components move to considerably higher energies. However, since the magnetic field orientation remains unchanged, as in the previous scenario, the P.A. will stay confined to at most $\left(45^{\circ}, 135^{\circ}\right)$.

For Mkn 421, due to the unchanged magnetic field, the higher-energy electrons take longer to cool than in the previous scenarios so that the flare duration is longer. Also, the electron spectral index remains nearly constant while the shock is present (Figure 12, left), so that $\Pi_{\max }$ will be nearly unchanged throughout the emission region. However, after the shock leaves a given zone, the spectrum hardens at lower energies while softening at higher energies. Since this effect acts extremely slowly and is very weak, its contribution to both luminosity and the polarization can only be seen during the post-peak phase. As a result, the polarization region dominates only because of its high luminosity. Another reason for the different polarization behavior with respect to the previous scenarios is that the polarization region is larger, containing more evolving 

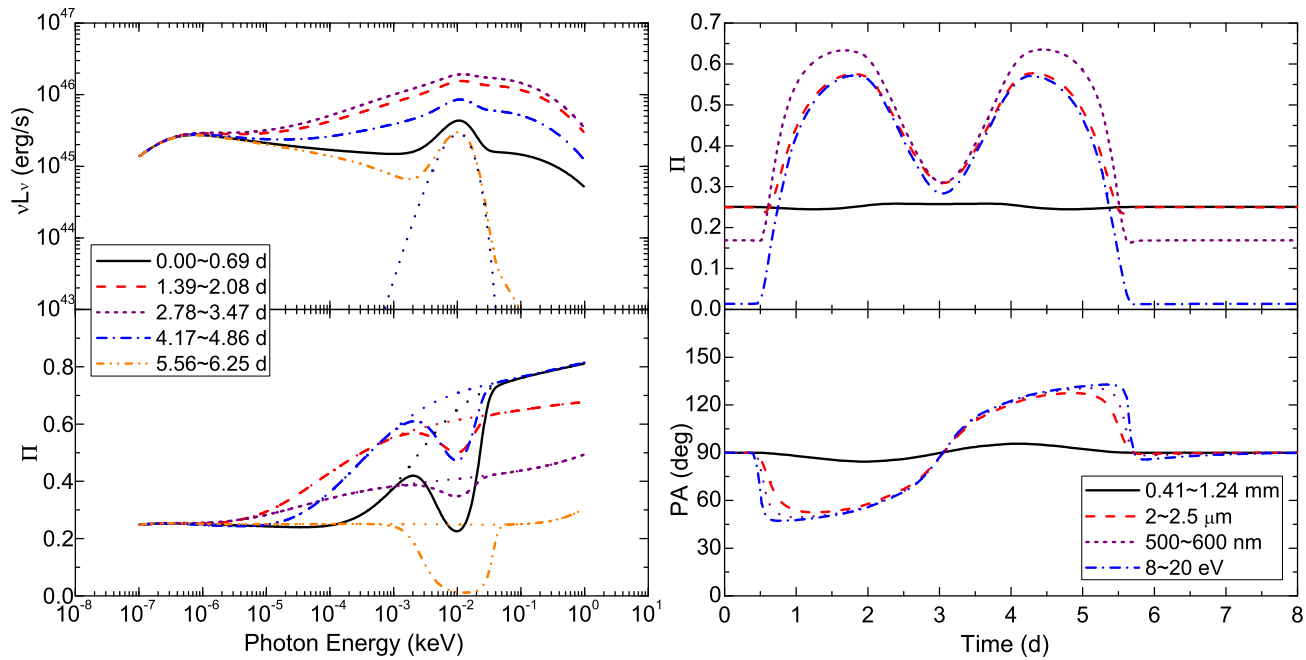

Figure 11. Scenario 3 (shortened acceleration timescale) for PKS 1510-089. Panels and line styles are as in Figure 9.

(A color version of this figure is available in the online journal.)

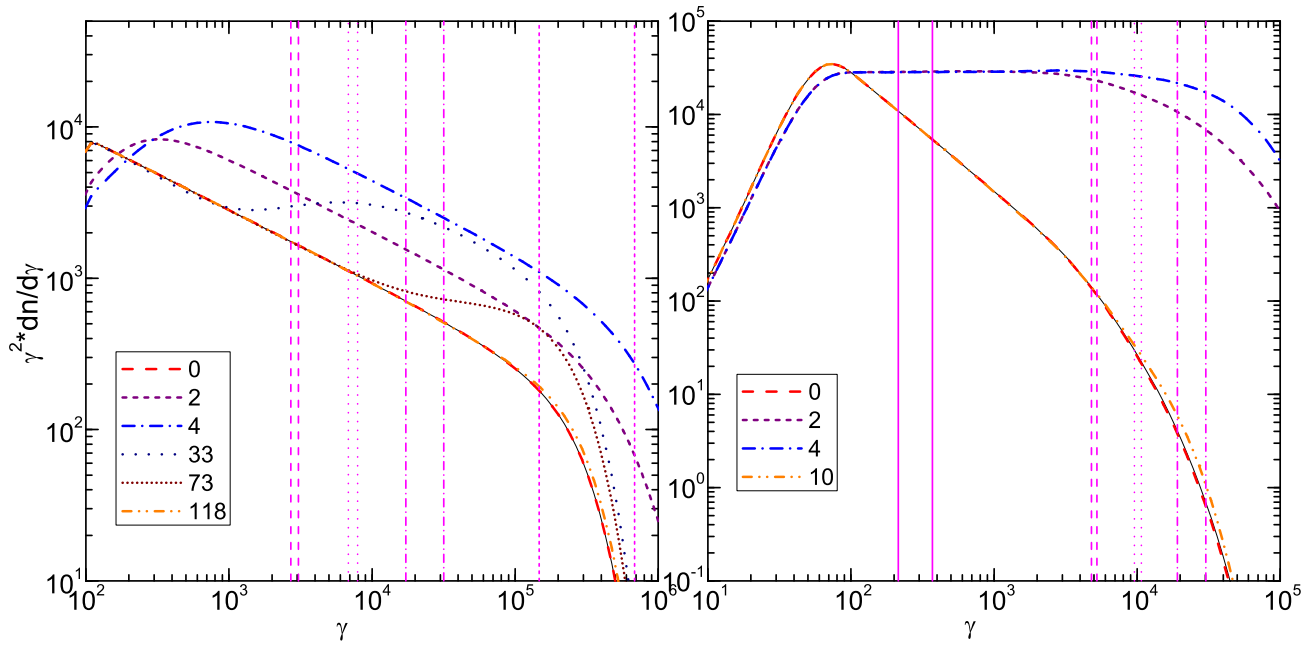

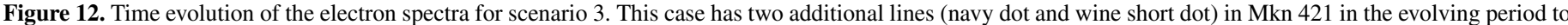

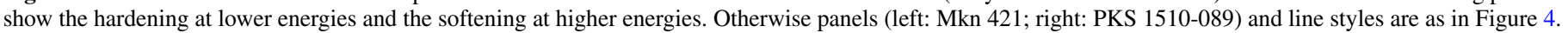
(A color version of this figure is available in the online journal.)

zones. This effect is especially strong after the flare peak, when much of the emission region has been affected by the shock. Consequently, the emission region is nearly equivalent to the initial state, except that all zones radiate with higher luminosity. Therefore, we see that the polarization percentage is lower overall than in the previous scenarios, and the pre-peak polarization percentage is higher than in the post-peak phase. Just like the light curves, also the polarization percentage takes longer to evolve at higher energies.

The situation for PKS 1510-089 is somewhat different. When the shock reaches a given zone, the shortened acceleration timescale results in a much harder spectrum, which will give lower $\Pi_{\max }$. After the shock leaves the zone, due to the strong EC cooling, the electron spectrum quickly evolves back to equilibrium (Figure 12, right). As a result, the polarization region is very narrow. However, a much more significant factor is that the flare-to-equilibrium luminosity ratio is very large in this case (Figure 11, left). Therefore, the contribution from background regions to the polarization is negligible. Hence, although $\Pi_{\max }$ is lower in the polarization region, this effect is compensated by the highly ordered magnetic field in the polarization region in the pre-peak $\left(\sim-45^{\circ}\right)$ and post-peak $\left(\sim-135^{\circ}\right)$ periods of the flare. The basin at the flare peak is, again, due to the axisymmetry of the polarization region. The P.A. shows similar features, achieving its minimum quickly at the beginning of the flare, gradually evolving to $90^{\circ}$ at the peak, then to maximum at the end of the flare and back to $90^{\circ}$ in equilibrium. There is one exception, however: at radio frequencies, the flare-to-equilibrium ratio is nearly 1 , thus we see both the polarization percentage and angle staying nearly constant.

\subsection{Injection of Particles}

In this scenario, the shock is assumed to continuously inject relativistic particles in the zones that it crosses (parameters for the injected electrons can be found in Table 1). The newly injected electrons will evolve and radiate immediately after the injection, in the same way as the original electrons in that zone. This scenario is similar to the previous one, except for the following differences.

First, in the case of Mkn 421, the newly injected electrons occupy an energy range not extending beyond the equilibrium electron distribution (see Figure 15, left). In particular, the flare electron spectrum will not extend to higher energies than the equilibrium distribution, and therefore the electron 

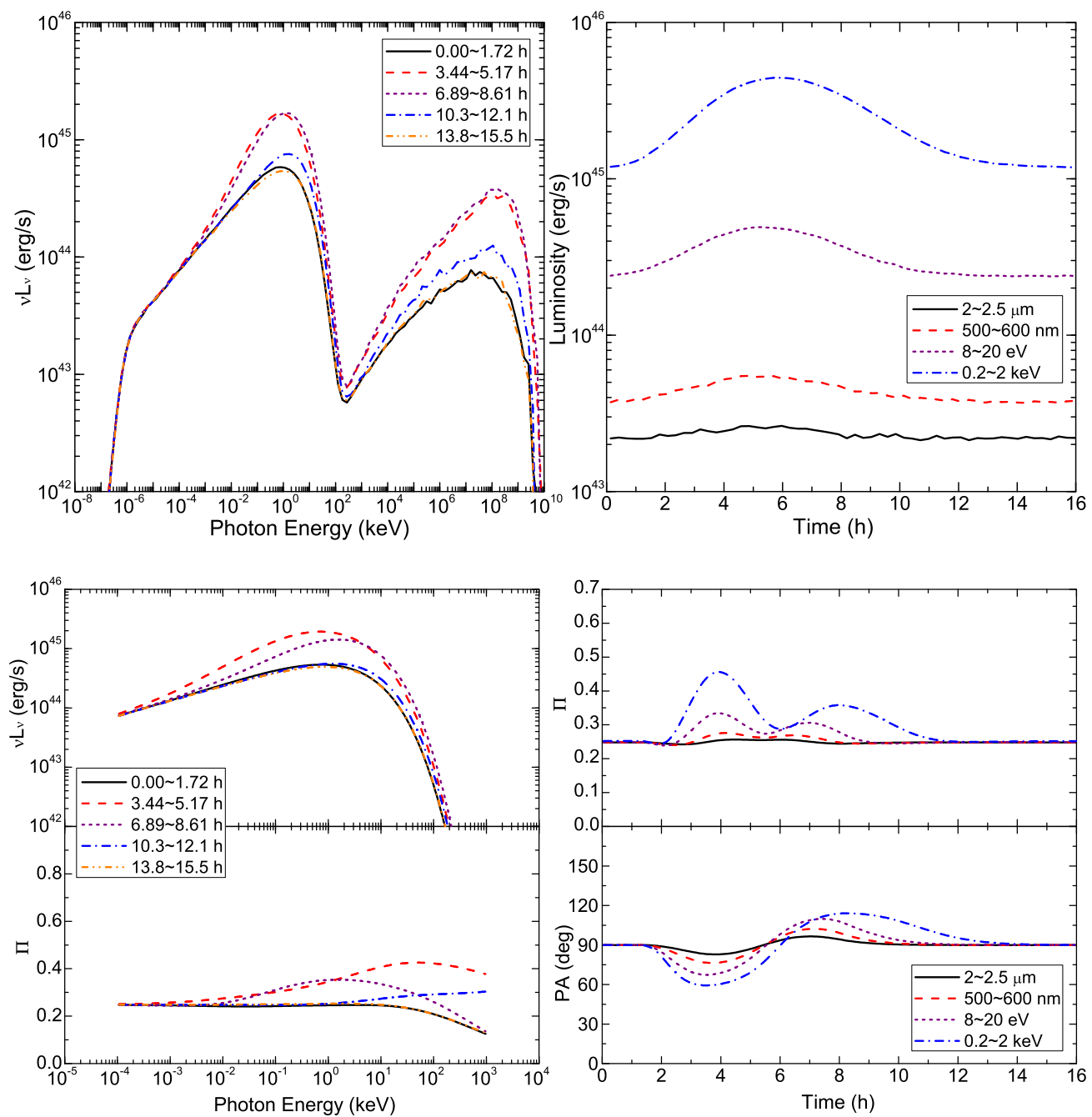

Figure 13. Scenario 4 (injection of additional high-energy electrons) for Mkn 421. Panels and line styles are as in Figure 3.

(A color version of this figure is available in the online journal.)

cooling timescales remain almost unaffected. Consequently, the X-ray flare in Mkn 421 again stops earlier (Figure 13, upper right). Second, although immediately after the injection the electron spectrum hardens, at the highest energies, it will become softer than the initial spectrum while the additional high-energy electrons cool off to lower energies, resulting in a higher $\Pi_{\max }$. However, at lower energies the flare electron spectrum will generally be harder than the equilibrium spectrum. Therefore, the polarization percentage in general increases at higher energies, but decreases at lower energies (Figures 13, lower left and 14, left). The same applies to PKS 1510-089, but we observe that the polarization percentage increases a little bit in radio but decreases in ultraviolet. The reason is that the radio has a little bit higher flare-to-equilibrium luminosity ratio than that in the previous scenario, while that for ultraviolet is lower, so that its polarization is contaminated more by the external photon field in the dusty torus. The P.A. swings follow similar patterns as in the previous scenario.

\section{DISCUSSION}

In Section 3, we have shown both the energy and the time dependencies of the synchrotron fluxes and polarization patterns in a generic shock-in-jet scenario for four different possible mechanisms through which a shock may result in synchrotron flaring behavior. We have chosen model parameter values that have been shown to be appropriate to reproduce SEDs and light curves of Mkn 421 and PKS 1510-089. However, there are still parameter degeneracies, and some of the geometric parameters, such as the ratio between $z$ and $r$, and the viewing angle, i.e., the direction of the LOS with respect to the jet axis, $\theta_{\mathrm{obs}}$, have been fixed without strict observational constraints. In this section, we will show that the choice of these parameters may have a nonnegligible influence on the predicted polarization patterns. Specifically, we use $\theta_{\text {obs }}$ as an example to discuss the geometric effect on the polarization.

\subsection{Dependence on the Viewing Angle}

Throughout Section 3, we have assumed that we are observing the blazar jet from the side $\left(\theta_{\mathrm{obs}}=90^{\circ}\right)$ in the co-moving frame, due to relativistic aberration. However, the relativistic beaming effects will be very similar for viewing angles that are a few degrees off this angle-in particular toward smaller viewing angles. Here we investigate the scenario 1 (for which we have shown that large P.A. rotations are naturally predicted) under two different viewing angles, $\theta_{\mathrm{obs}}$, namely $60^{\circ}$ and $80^{\circ}$, to illustrate this geometric effect on the polarization. Although $\theta_{\text {obs }}$ can in principle be greater than $90^{\circ}$, it is unlikely that $\theta_{\mathrm{obs}}$ is much greater than $90^{\circ}$, as we expect statistically to observe 

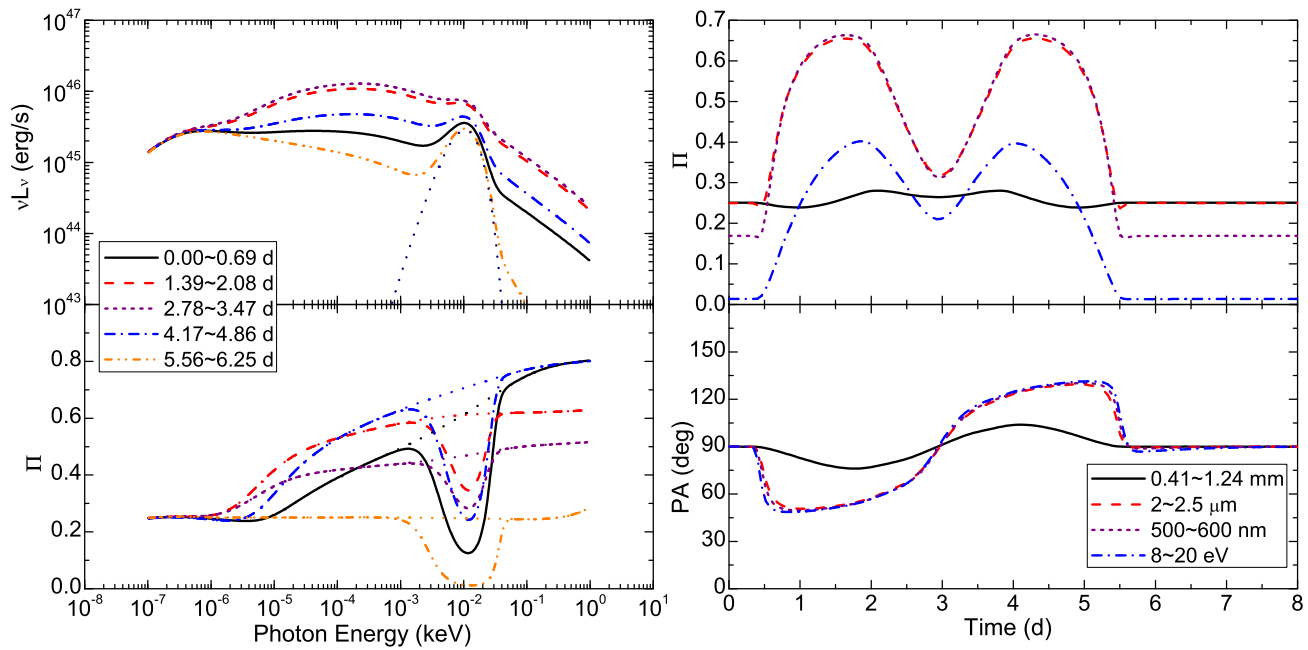

Figure 14. Scenario 4 (injection of additional high-energy electrons) for PKS 1510-089. Panels and line styles are as in Figure 9.

(A color version of this figure is available in the online journal.)
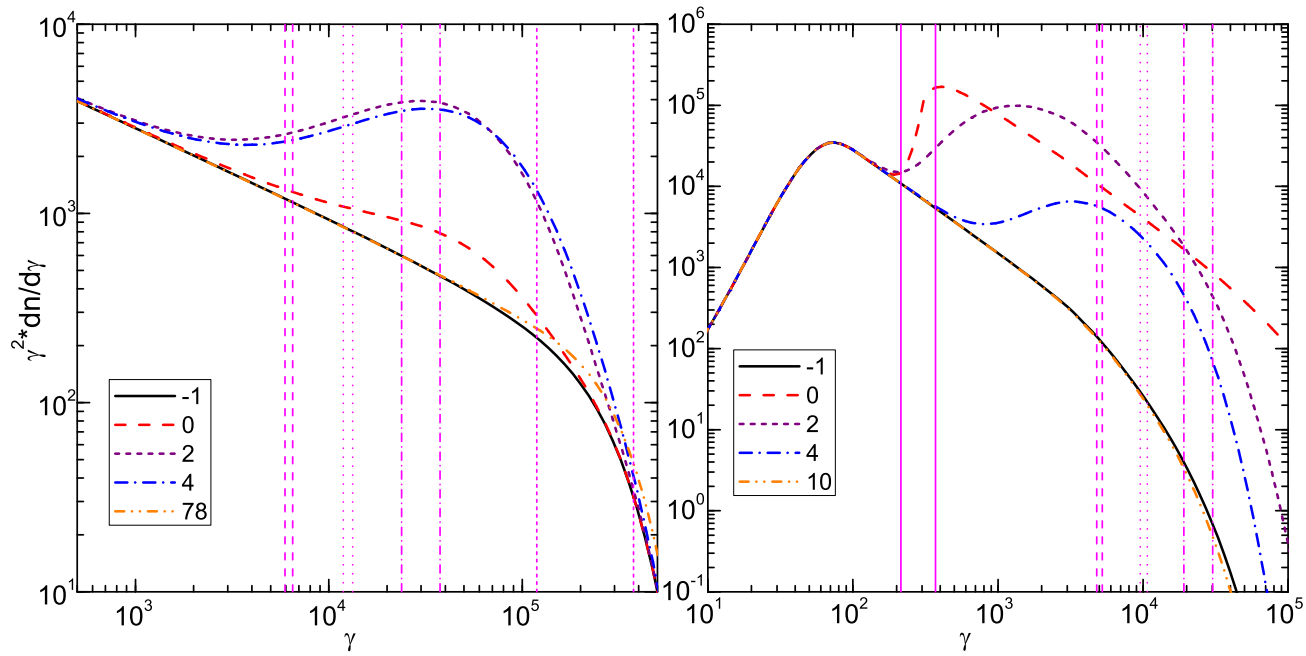

Figure 15. Time evolution of the electron spectra for scenario 4. Notice in this case the spectra at pre-shock equilibrium and at the onset of the shock are different, as the electrons are injected. Panels (left: Mkn 421; right: PKS 1510-089) and line styles are as in Figure 4.

(A color version of this figure is available in the online journal.)

most blazar jets from within the relativistic beaming cone, given by $\theta_{\text {obs }}^{*} \lesssim 1 / \Gamma$ in the observer's frame, which corresponds to $\theta_{\text {obs }} \lesssim 90^{\circ}$ in the comoving frame.

With a $\theta_{\text {obs }}$ value differing from $90^{\circ}$, the polarization region will be a bit smaller and located differently. $B_{y_{0}}$ is as well not affected, but the major change here is $B_{z_{0}}$. We observe that $B_{\perp}$, as well as the effective poloidal component $B_{z_{0}}$, is stronger in $-y$ while weaker in $+y$ (Figure 16). Thus the axisymmetry discussed in Section 3 is invalid. As a result, the emission from $-y$, where $B_{\perp}$ has a relatively stronger poloidal contribution, will dominate over the emission from $+y$, where $B_{\perp}$ has a dominant toroidal contribution. However, in the initial state, although $B_{z_{0}}$ is stronger near the $-y$ axis, it is much weaker near the $+y$ axis and near the $x= \pm r_{\max }$ boundaries, thus the polarization due to $B_{z_{0}}$ is overall weaker than that in the $\theta_{\text {obs }}=90^{\circ}$ case. Therefore, at the pre-flare and post-flare equilibrium states, the P.A. has the same value as before, while the polarization percentage is lower.

During the flare, however, unlike in Section 3.1 where amplification of $B_{\phi}$ leads to a dramatic increase in $B_{y_{0}}$, this time it also contributes to $B_{z_{0}}$, especially at the flare peak (Figure 16).
As a result, the polarization due to $B_{y_{0}}$ will be balanced out more by that from $B_{z_{0}}$. Hence the polarization caused by the toroidal magnetic-field component takes longer to reach maximum after its dominance over the original polarization due to $B_{z_{0}}$, so that the dip in the polarization percentage versus time is wider (Figure 17), and the polarization percentage is generally lower with smaller $\theta_{\text {obs }}$ (obviously, the net polarization goes to zero in the limit $\theta_{\text {obs }} \rightarrow 0^{\circ}$ ). This effect is particularly strong in the $\theta_{\text {obs }}=60^{\circ}$ case shown in Figure 17 (upper), where we observe that in the pre-peak and the post-peak flaring state, there are two small dips in the polarization percentage with corresponding fluctuations in the P.A. This is because the toroidal component is less dominant: at the beginning of the flare, the polarization region is small, but the toroidal component is highly ordered and is oriented in the $y_{0}$ direction (Figure 16). This will give strong polarization in P.A. $=180^{\circ}$, which will quickly cancel out the background P.A. $=270^{\circ}$ polarization and dominate. However, when the polarization region moves closer to the center, $B_{z_{0}}$ will increase on the $-y$ side, which dominates the emission; meanwhile, the background region will be dominated by emission from the central and $-x$ regions, which will have 


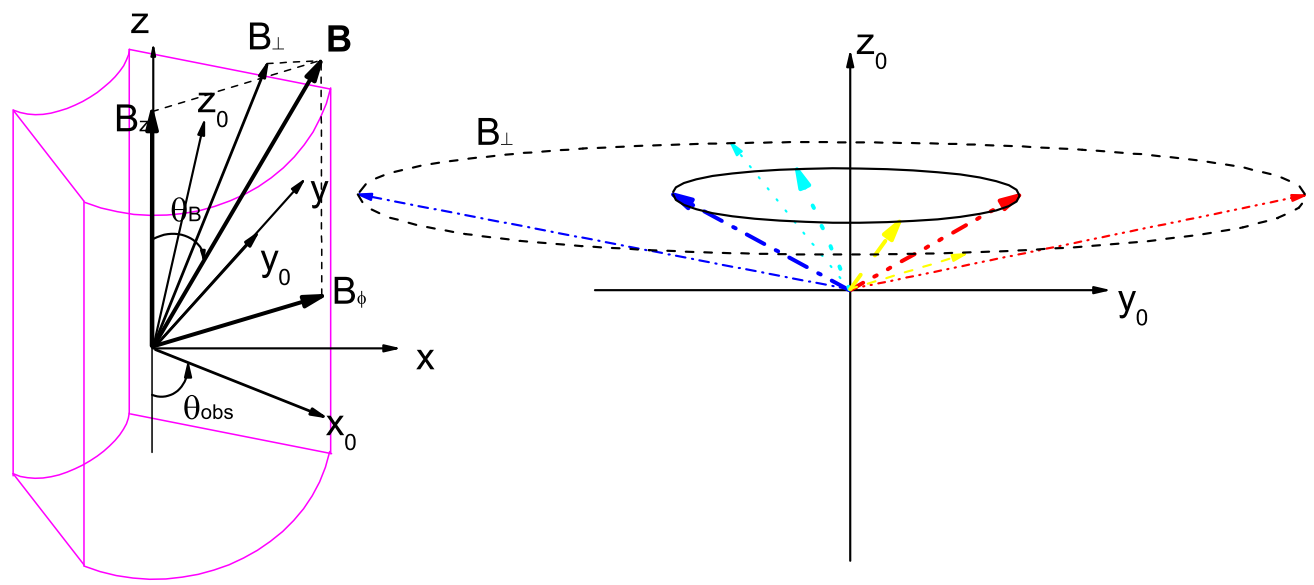

Figure 16. Similar to Figure 6 but for $\theta_{\mathrm{obs}}=60^{\circ}$. Here the yellow $(+y)$ and cyan $(-y)$ regions are not symmetric, hence the strengths of $B_{x_{0}}$ and $B_{y_{0}}$ change accordingly.

(A color version of this figure is available in the online journal.)
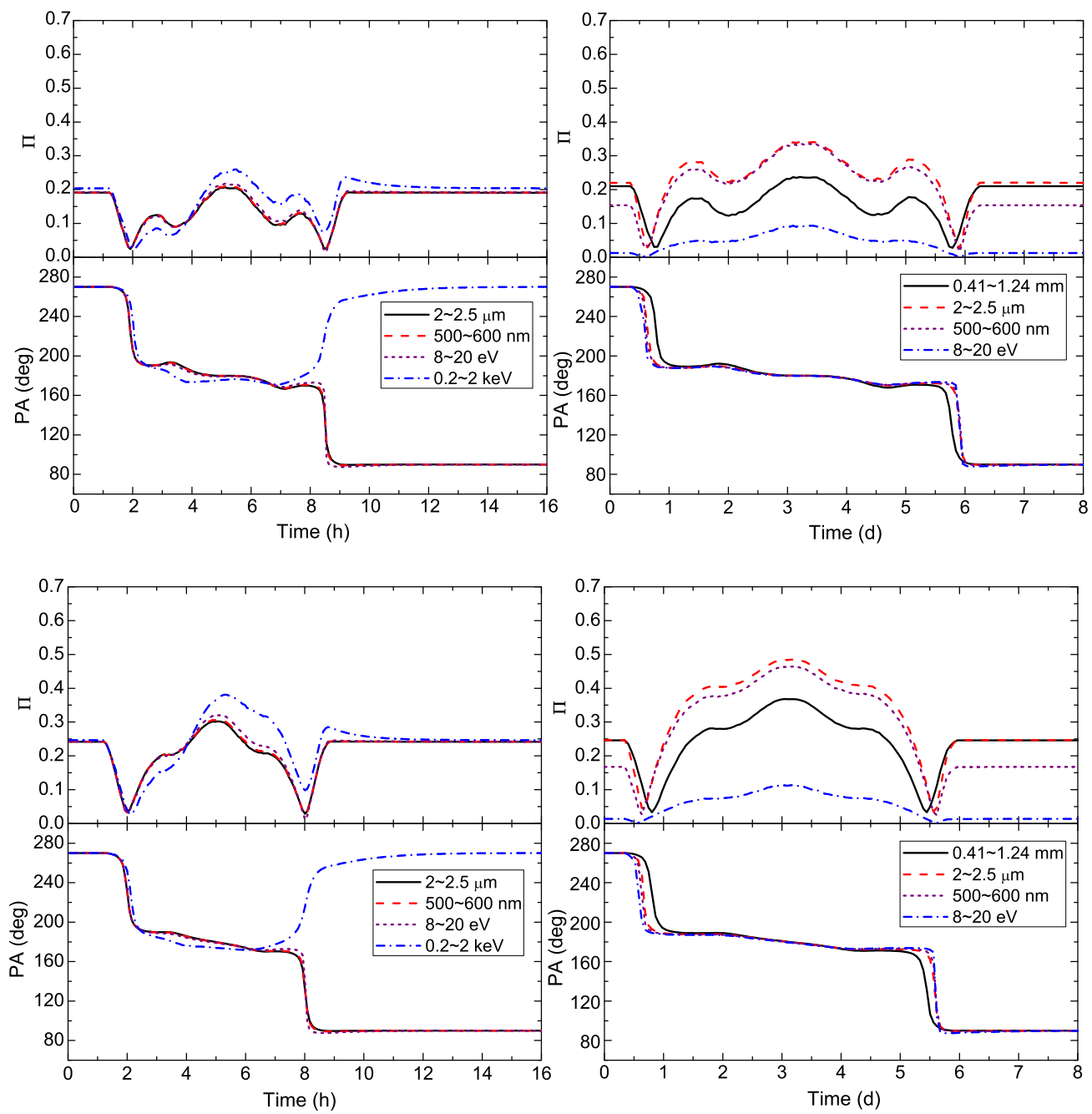

Figure 17. Polarization vs. time plots for flaring scenario 1, for different viewing angles, for comparison with Figures 3 (lower) and 7 (lower). Left column: Mkn 421 . Right column: PKS 1510-089. Top row: $\theta_{\mathrm{obs}}=60^{\circ}$. Bottom row: $\theta_{\mathrm{obs}}=80^{\circ}$.

(A color version of this figure is available in the online journal.)

stronger poloidal polarization than produced in the $+x$ region in the initial state. Hence, the poloidal contribution to the polarization increases. When the polarization region moves to the flare-peak position, however, the central region is affected by the shock. Although $B_{z_{0}}$ will become even stronger near the $-y$ axis, $B_{\perp}$ in its neighborhood has a stronger $B_{y_{0}}$ component. Since the polarization region extends to neighboring regions, the polarization due to $B_{y_{0}}$ will regain its dominance. The post- 
peak and the post-flare equilibrium evolve in the same way, as the polarization region is symmetric in the time domain, except for slight differences in X-ray.

\section{SUMMARY AND CONCLUSIONS}

In this paper, we have presented a detailed analysis of timeand energy-dependent synchrotron polarization signatures in a shock-in-jet model for $\gamma$-ray blazars. Our calculations employ a full 3D radiation transfer code, assuming a helical magnetic field throughout the jet, carefully taking into account light-traveltime and all other relevant geometric effects. We considered several possible mechanisms through which a relativistic shock propagating through the jet may affect the jet plasma to produce a synchrotron and high-energy flare. Among the scenarios investigated, we found that a compression of the magnetic field, increasing the toroidal field component and thereby changing the direction of the magnetic field in the region affected by the shock, leads to correlated synchrotron +SSC flaring, associated with substantial variability in the synchrotron polarization percentage and position angle. Most importantly, this scenario naturally explains large P.A. rotations by $\gtrsim 180^{\circ}$, as observed in connection with $\gamma$-ray flares in several blazars. In particular, we have refuted the claim (e.g., Abdo et al. 2010b) that pattern propagation through an axisymmetric, straight jet cannot produce large P.A. swings and rotations.

Our model predicts correlated synchrotron (optical/UV/ $\mathrm{X}$-ray) flaring activity associated with drastic changes in the degree of polarization and polarization angle swings by multiples of $180^{\circ}$. If the magnetic-field change occurs as abruptly as assumed in our simulations, we expect that such rotations might occur in steps of individual $\sim 90^{\circ}$ swings on short timescales. The associated timescale of such polarization-angle steps would then be a measure of the transverse light crossing time through the emission region. In SSC-dominated blazars and/or if the shock presumably responsible for the magnetic-field change is also leading to a substantial increase in the particle-acceleration efficiency (shortened $t_{\text {acc }}$ ), such synchrotron flares are expected to be correlated with $\gamma$-ray flaring activity.

We note that our choice of a purely helical magnetic field maximizes the expected degree of polarization for any given value of $\theta_{B}$. A chaotic magnetic-field component would add an effectively unpolarized emission component to the synchrotron emission and would thereby diminish the resulting total polarization.

Alternative models to explain polarization variability and P.A. rotations, include a helical guiding magnetic field, which forces plasmoids to move along helical paths (Villata \& Raiteri 1999), and the TEMZ model by Marscher (2014). Abdo et al. (2010b) have suggested that P.A. swings correlated with $\gamma$-ray flaring activity may result when a shock or other disturbance propagates along a curved (helical) jet. In the course of the propagation along a curved trajectory, the observer's viewing angle with respect to the co-moving magnetic field in the active region changes, leading to possible P.A. swings. While such an explanation seems plausible on geometric grounds, no quantitative analysis of the resulting, correlated synchrotron and highenergy flux and polarization features has been presented for such a model, and there is currently no evidence (e.g., from observations or from MHD simulations) that blazar jets are guided by sufficiently strong, helical magnetic fields that would be able to guide relativistic pattern propagation along helical trajectories. Our analysis in this paper has demonstrated that LTTEs lead to much more complicated time-dependent polarization features than predicted by purely geometric considerations that neglect LTTEs.

By the stochastic nature of the TEMZ model (Marscher 2014), it predicts generally asymmetrical light curves and random polarization patterns that do only occasionally (by coincidence) lead to large-angle P.A. swings, which will generally not be correlated with pronounced flaring activity at higher energies. Observed polarization angle changes do, in fact, often appear stochastic in nature, and even the polarization-swing event reported in Abdo et al. (2010b) showed signs of nonunidirectional P.A. changes and may therefore be interpreted by a stochastic model such as the TEMZ model. The TEMZ code of Marscher (2014) takes into account SSC scattering (and its influence on electron cooling) only with seed photons from the central mach disk. Therefore, it is well applicable for blazars in which $\gamma$-ray emission and electron cooling are dominated by Comptonization of external radiation fields, which appears to be the case in low-frequency peaked blazars (FSRQs, LBLs), but not for HBLs like Mrk 421, in which the $\gamma$-ray emission is well modeled as being dominated by SSC radiation.

The strength of the P.A. rotation model presented here is that it very naturally explains large P.A. rotations, correlated with $\gamma$-ray flaring events, without the need for non-axisymmetric jet features. It is supported by observations of large-angle, unidirectional polarization swings, e.g., in 3C279 (Kiehlmann et al. 2013), which suggest that such features are unlikely to be caused by a stochastic process, but are likely the result of preferentially ordered structures. For these reasons, we prefer our quite natural explanation of P.A. swings correlated with synchrotron and high-energy flares, resulting from light-travel-time effects in a shock-in-jet model in a straight, axisymmetric jet embedded in a helical magnetic field.

We thank the anonymous referee for a careful review of the paper and helpful suggestions to improve the clarity of the manuscript, and Alan Marscher for valuable discussions and comments. This work was supported by NASA through Fermi Guest Investigator Grant no. NNX12AP20G. H.Z. is supported by the LANL/LDRD program and by DoE/Office of Fusion Energy Science through CMSO. X.C. acknowledges support by the Helmholtz Alliance for Astroparticle Physics HAP funded by the Initiative and Networking Fund of the Helmholtz Association. X.C. gratefully acknowledges the support during his visit to LANL when this work was started. M.B. acknowledges support by the South African Research Chairs Initiative of the Department of Science and Technology and the National Research Foundation of South Africa. Simulations were conducted on LANL's Institutional Computing machines.

\section{REFERENCES}

Abdo, A. A., Ackermann, M., Agudo, I., et al. 2010a, ApJ, 721, 1425

Abdo, A. A., Ackermann, M., Ajello, M., et al. 2010b, Natur, 463, 919

Aharonian, F. A., Akhperjanian, A. G., Bazer-Bachi, A. R., et al. 2007, ApJL, 664, L71

Albert, J., Aliu, E., Anderhub, H., et al. 2007, ApJ, 669, 862

Aloy, M.-A., Gomez, J.-L., Ibáñez, J.-M., Martí, J.-M., \& Müller, E. 2000, ApJL, 528, L85

Böttcher, M. 2007, ApSS, 309, 95

Böttcher, M., \& Reimer, A. 2012, in Relativistic Jets from Active Galactic Nuclei, ed. M. Böttcher, D. Harris, \& H. Krawczynski (Berlin: Wiley-VCH), 39

Chen, X., Fossati, G., Böttcher, M., \& Liang, E. 2012, MNRAS, 424, 789

Chen, X., Fossati, G., Liang, E., \& Böttcher, M. 2011, MNRAS, 416, 2368

Contopoulos, J. 1994, ApJ, 432, 508

D’Ammando, F., Raiteri, C. M., Villata, M., et al. 2011, A\&A, 529, A145 
D’Arcangelo, F. D., Marscher, A. P., Jorstad, S. G., et al. 2007, ApJL, 659, L107 Fossati, G., Buckley, J. H., Bond, I. H., et al. 2008, ApJ, 677, 906

Ikejiri, Y., Uemura, M., Sasada, M., et al. 2011, PASJ, 63, 639

Kiehlmann, S., Savolainen, T., Jorstad, S. G., et al. 2013, in EPJ Web of Conferences, Vol. 61, The Innermost Regions of Relativistic Jets and Their Magnetic Fields, Granada, Spain, ed. J. Gómez (Les Ulis, France: EDP Sciences), 06003

Krawczynski, H., Böttcher, M., \& Reimer, A. 2012, in Relativistic Jets from Active Galactic Nuclei, ed. M. Böttcher, D. Harris, \& H. Krawczynski (Berlin: Wiley-VCH), 215

Laing, R. 1980, MNRAS, 193, 439
Lyutikov, M., Pariev, V. I., \& Gabuzda, D. C. 2005, MNRAS, 360, 869

Marscher, A. P. 2014, ApJ, 780, 87

Marscher, A. P., Jorstad, S. G., D' Arcangelo, F. D., et al. 2008, Natur, 452, 966

Marscher, A. P., Jorstad, S. G., Larionov, V. M., et al. 2010, ApJL, 710, L126

Pushkarev, A. B., Gabuzda, D. C., Vetukhnovskaya, Yu. N., \& Yakimov, V. E. 2005, MNRAS, 356, 859

Rybicki, G. B., \& Lightman, A. P. 1985, Radiative processes in Astrophysics (Berlin: Wiley-VCH)

Villata, M., \& Raiteri, C. M. 1999, A\&A, 347, 30

Westfold, K. C. 1959, ApJ, 130, 241

Zhang, H., \& Böttcher, M. 2013, ApJ, 774, 18 